\title{
Algebraic entropy, symmetries and linearization of quad equations consistent on the cube.
}

\author{
G. Gubbiotti* C. Scimiterna ${ }^{\dagger} \quad$ D. Levi ${ }^{\ddagger}$ \\ Dipartimento di Matematica e Fisica, Universita' degli Studi Roma Tre, \\ e Sezione INFN di Roma Tre, \\ Via della Vasca Navale 84, 00146 Roma (Italy)
}

September 5, 2018

\begin{abstract}
We discuss the non autonomous nonlinear partial difference equations belonging to Boll classification of quad graph equations consistent around the cube. We show how starting from the compatible equations on a cell we can construct the lattice equations, its Bäcklund transformations and Lax pairs. By carrying out the algebraic entropy calculations we show that the $H^{4}$ trapezoidal and the $H^{6}$ families are linearizable and in a few examples we show how we can effectively linearize them.
\end{abstract}

\section{Introduction}

In recent years the integrability criteria denoted Consistency Around the Cube $(\mathrm{CAC})$ has been a source of many results in the classification of nonlinear difference equations on a quad graph. Its importance relays in the fact that provides Bäcklund transforms $7,11,24,25$ and as a consequence its zero curvature representation or Lax pairs. As it is well known 32] Lax pairs and Bäcklund transforms are associated to both linearizable and integrable equations. While in the integrable case the solution of the Lax pair provides genuine nontrivial solutions [14] in the linearizable case the Lax pair is fake [12,13, 19]

The first attempt to carry out a classification of partial difference equations using the CAC condition has been presented in 1 assuming that the equations on all faces of the cube had the same form. The result is a class of discrete equations whose basic building blocks are equations on quadrilaterals of the type

$$
A\left(x, x_{1}, x_{2}, x_{12} ; \alpha_{1}, \alpha_{2}\right)=0,
$$

where the four fields $x, x_{1}, x_{2}$ and $x_{12} \in \mathbb{C}$ are assigned to the four vertexes of a quadrilateral and the parameters $\alpha_{i} \in \mathbb{C}, i=1,2$ to its edges, see Fig. 1 (later on, in (1.3), the points $x, x_{1}, x_{2}$ and $x_{12}$ will be denoted by $x_{1}, x_{2}, x_{3}$ and $x_{4}$ ). In the notation of (1.1), if the quadrilateral is constructed from an origin on

\footnotetext{
*e-mail: gubbiotti@mat.uniroma3.it

†e-mail: scimiterna@fis.uniroma3.it

‡e-mail: decio.levi@roma3.infn.it
} 


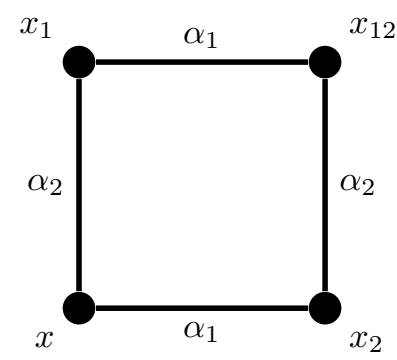

Figure 1: Quad-graph

which the field $x$ is assigned introducing two independent directions 1 and 2; the subscript 1 and 2 denote the field in the vertex shifted by $\alpha_{1}$ and $\alpha_{2}$ along the direction 1 and 2 from the origin, while 12 refers to the field in the remaining vertex of the quadrilateral. Moreover the function $A\left(x, x_{1}, x_{2}, x_{12} ; \alpha_{1}, \alpha_{2}\right)$ is assumed to be affine linear in each argument (multilinearity) with coefficients depending on the two edge parameters and invariant under the discrete group $D_{4}$ of the symmetries of the square

$$
\begin{aligned}
A\left(x, x_{1}, x_{2}, x_{12} ; \alpha_{1}, \alpha_{2}\right) & =\varepsilon A\left(x, x_{2}, x_{1}, x_{12} ; \alpha_{2}, \alpha_{1}\right) \\
& =\sigma A\left(x_{1}, x, x_{12}, x_{2} ; \alpha_{1}, \alpha_{2}\right), \quad(\varepsilon, \sigma)= \pm 1 .
\end{aligned}
$$

A last simplifying hypothesis is the so called tetrahedron property which amounts

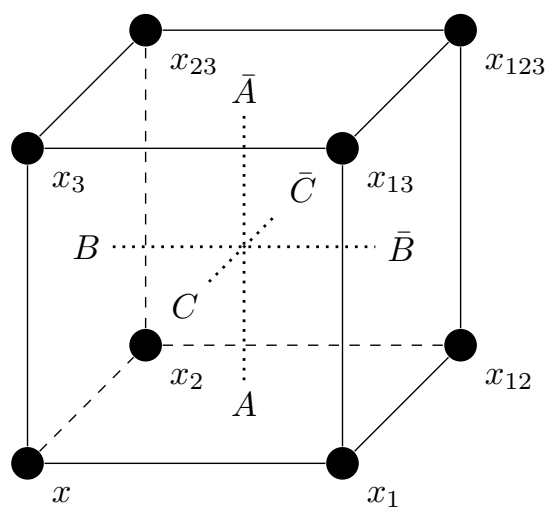

Figure 2: Equations on a Cube

to require that, having introduced a third independent direction 3 and a point $x_{3}$ constructed in the direction 3 at a distance $\alpha_{3}$ from the origin $x$, the function $x_{123} \doteq x_{123}\left(x, x_{1}, x_{2}, x_{3} ; \alpha_{1}, \alpha_{2}, \alpha_{3}\right)$ does not depend on $x$. The result of the classification (up to a common Möbius transformation of the field variables and point transformations of edge parameters) is given by two lists of equations, $H$ and $Q$, for a total of seven consistent systems:

$$
\begin{aligned}
H_{1}: & \left(x-x_{12}\right)\left(x_{2}-x_{1}\right)-\alpha_{1}+\alpha_{2}=0, \\
H_{2}: & \left(x-x_{12}\right)\left(x_{2}-x_{1}\right)+\left(\alpha_{2}-\alpha_{1}\right)\left(x+x_{2}+x_{1}+x_{1,2}\right)- \\
& \quad-\alpha_{1}^{2}+\alpha_{2}^{2}=0,
\end{aligned}
$$




$$
\begin{gathered}
H_{3}: \alpha_{1}\left(x x_{2}+x_{1} x_{12}\right)-\alpha_{2}\left(x x_{1}+x_{2} x_{12}\right)+\delta\left(\alpha_{1}^{2}-\alpha_{2}^{2}\right)=0 \\
Q_{1}: \alpha_{1}\left(x-x_{1}\right)\left(x_{2}-x_{12}\right)-\alpha_{2}\left(x-x_{2}\right)\left(x_{1}-x_{12}\right)+ \\
\quad+\delta^{2} \alpha_{1} \alpha_{2}\left(\alpha_{1}-\alpha_{2}\right)=0 \\
Q_{2}: \alpha_{1}\left(x-x_{1}\right)\left(x_{2}-x_{12}\right)-\alpha_{2}\left(x-x_{2}\right)\left(x_{1}-x_{12}\right)+ \\
\quad+\alpha_{1} \alpha_{2}\left(\alpha_{1}-\alpha_{2}\right)\left(x+x_{2}+x_{1}+x_{12}\right)- \\
\quad-\alpha_{1} \alpha_{2}\left(\alpha_{1}-\alpha_{2}\right)\left(\alpha_{1}^{2}-\alpha_{1} \alpha_{2}+\alpha_{2}^{2}\right)=0 \\
Q_{3}:\left(\alpha_{2}^{2}-\alpha_{1}^{2}\right)\left(x x_{12}+x_{2} x_{1}\right)+\alpha_{2}\left(\alpha_{1}^{2}-1\right)\left(x x_{2}+\right. \\
\left.\quad+x_{1} x_{12}\right)-\alpha_{1}\left(\alpha_{2}^{2}-1\right)\left(x x_{1}+x_{12} x_{12}\right)- \\
\quad-\frac{\delta^{2}\left(\alpha_{1}^{2}-\alpha_{2}^{2}\right)\left(\alpha_{1}^{2}-1\right)\left(\alpha_{2}^{2}-1\right)}{4 \alpha_{1} \alpha_{2}}=0 \\
Q_{4}: a_{0} x_{2} x_{1} x_{12}+a_{1}\left(x x_{2} x_{1}+x_{2} x_{1} x_{12}+x_{1} x_{12} x+\right. \\
\left.\quad+x_{12} x x_{2}\right)+a_{2}\left(x x_{12}+x_{2} x_{1}\right)+\bar{a}_{2}\left(x x_{2}+\right. \\
\left.\quad+x_{1} x_{12}\right)+\tilde{a}_{2}\left(x x_{1}+x_{2} x_{12}\right)+a_{3}\left(x+x_{2}+x_{1}+\right. \\
\left.\quad+x_{12}\right)+a_{4}=0
\end{gathered}
$$

where the constants $a_{0}, a_{1}, a_{2}, \bar{a}_{2}, \tilde{a}_{2}, a_{3}$ and $a_{4}$ are well defined elliptic expressions in terms of $\alpha_{1}$ and $\alpha_{2}$ [1]. In (1.2) the $Q$-type equations are those where all of the six accompanying biquadratics, defined by

$$
A^{i, j} \equiv A^{i, j}\left(x_{i}, x_{j}\right)=A_{, x_{m}} A_{, x_{n}}-A A_{, x_{m} x_{n}},
$$

where $\{m, n\}$ is the complement of $\{i, j\}$ in $\{1,2,3,4\}$, are non degenerate, i.e. all different, while in the $H$-type equations some of the biquadratics are degenerate. In Fig. $2 \bar{A}$ represents a copy of the equation $A$ (one of the seven equations (1.2a) ) with $x$ substituted by $x_{3}, x_{1}$ by $x_{13}, x_{2}$ by $x_{23}$ and $x_{12}$ by $x_{123}$. On the faces $B$ and $\bar{B}$ we have a relation between a solution of $A$ and one of $\bar{A}$, i.e. an auto-Bäcklund transformation for $A$. By going over to projective space the auto-Bäcklund transformation will provide the Lax pair.

In 2] the authors considered a more general perspective in the classification problem. They assumed that the faces of the consistency cube $A, B, C$ and $\bar{A}$, $\bar{B}$ and $\bar{C}$ could carry a priori different quad equations without assuming either the $D_{4}$ symmetry or the tetrahedron property. They considered six-tuples of (a priori different) quad equations assigned to the faces of a $3 \mathrm{D}$ cube:

$$
\begin{array}{lll}
A\left(x, x_{1}, x_{2}, x_{12} ; \alpha_{1}, \alpha_{2}\right)=0, & \bar{A}\left(x_{3}, x_{13}, x_{23}, x_{123} ; \alpha_{1}, \alpha_{2}\right)=0, \\
B\left(x, x_{2}, x_{3}, x_{23} ; \alpha_{3}, \alpha_{2}\right)=0, & \bar{B}\left(x_{1}, x_{12}, x_{13}, x_{123} ; \alpha_{3}, \alpha_{2}\right)=0, \\
C\left(x, x_{1}, x_{3}, x_{13} ; \alpha_{1}, \alpha_{3}\right)=0, & \bar{C}\left(x_{2}, x_{12}, x_{23}, x_{123} ; \alpha_{1}, \alpha_{3}\right)=0,
\end{array}
$$

see Fig. 2. Such a six-tuple is defined to be $3 D$ consistent if, for arbitrary initial data $x, x_{1}, x_{2}$ and $x_{3}$, the three values for $x_{123}$ (calculated by using $\bar{A}=0, \bar{B}=0$ and $\bar{C}=0$ ) coincide. As a result in 2 they reobtained the $Q$-type equations of [1] and some new quad equations of type $H$ which turn out to be deformations of those present up above (1.2a 1.2c).

In [8 10], Boll, starting from [2], classified all the consistent equations on the quad graph possessing the tetrahedron property without any other additional assumption. The results were summarized by Boll in [10] in a set of theorems, from Theorem 3.9 to Theorem 3.14, listing all the consistent six-tuples configurations (1.4) up to $(\mathrm{Möb})^{8}$, the group of independent Möbius transformations 
of the eight fields on the vertexes of the consistency three dimensional cube, see Fig 2. All these equations fall into three disjoint families: $Q$-type (no degenerate biquadratic), $H^{4}$-type (four biquadratics are degenerate) and $H^{6}$-type (all of the six biquadratics are degenerate).

It's worth emphasizing that the classification results hold locally, i.e. the equations are valid on a single quadrilateral cell or on a single cube. The non secondary problem which has been solved is the embedding of the single cell/single cube equations in a $2 D / 3 D$ lattice, so as to preserve the $3 D$ consistency. This was discussed in [2] introducing the concept of Black-White (BW) lattice. To get the lattice equations one needs to embed (1.4) into a $\mathbb{Z}^{2}$ lattice with an elementary cell of dimension greater than one. In such a case the generic equation on a quad graph equation $Q\left(x, x_{1}, x_{2}, x_{12} ; \alpha_{1}, \alpha_{2}\right)=0$ is extended to a lattice and the lattice equation will have Lax pair and Bäcklund transformation. To do so, following [8], one reflects the square with respect to the normal to its right and top sides and then complete a $2 \times 2$ lattice by reflecting again one of the obtained equation with respect to the other direction 1 . Such a procedure is graphically described in Fig. 3, and at the level of the quad equation this corresponds to constructing the three equations obtained from $Q=Q\left(x, x_{1}, x_{2}, x_{12} ; \alpha_{1}, \alpha_{2}\right)=0$ by flipping its fields:

$$
\begin{aligned}
Q & =Q\left(x, x_{1}, x_{2}, x_{12}, \alpha_{1}, \alpha_{2}\right)=0, \\
\mid Q & =Q\left(x_{1}, x, x_{12}, x_{2}, \alpha_{1}, \alpha_{2}\right)=0, \\
\underline{Q} & =Q\left(x_{2}, x_{12}, x, x_{1}, \alpha_{1}, \alpha_{2}\right)=0, \\
\underline{Q} & =Q\left(x_{12}, x_{2}, x_{1}, x, \alpha_{1}, \alpha_{2}\right)=0 .
\end{aligned}
$$

By paving the whole $\mathbb{Z}^{2}$ with such equations we get a partial difference equation, which we can in principle study with the known methods. Since a priori $Q \neq$ $|Q \neq Q \neq| Q$ the obtained lattice will be a four stripe lattice, i.e. an extension of the BW lattice considered for example in [23, 31.

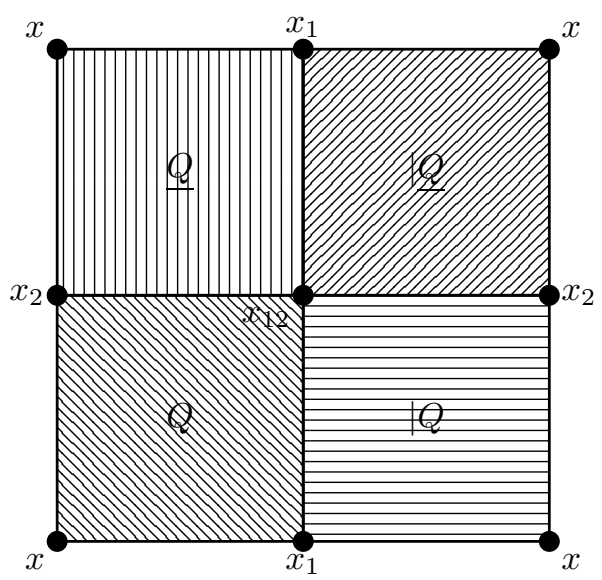

Figure 3: The "four colors" lattice

Let us notice that if the quad-equation $Q$ possess the symmetries of the

\footnotetext{
${ }^{1}$ Let us note that, whatsoever side we reflect, the result of the last reflection is the same.
} 
square, i.e. it is invariant under the action of $D_{4}$ one has:

$$
Q=|Q=\underline{Q}=| \underline{Q}
$$

implying that the elementary cell is actually of dimension one, and one falls into the case of the ABS classification. Beside the symmetry group of the square, $D_{4}$ [1] there are two others relevant discrete symmetries for quad equations (1.1). One is the rhombic symmetry, which holds when:

$$
\begin{aligned}
Q\left(x, x_{1}, x_{2}, x_{12}, \alpha_{1}, \alpha_{2}\right) & =\sigma Q\left(x, x_{2}, x_{1}, x_{12}, \alpha_{2}, \alpha_{1}\right) \\
& =\varepsilon Q\left(x_{12}, x_{1}, x_{2}, x, \alpha_{2}, \alpha_{1}\right), \quad(\sigma, \varepsilon) \in \pm 1
\end{aligned}
$$

Equations with rhombic symmetries have been introduced and classified in [2]. From their explicit form it is possible to show that they have the property:

$$
Q=|\underline{Q}, \quad \underline{Q}=| Q .
$$

The other kind of relevant discrete symmetry for quad equations is the trapezoidal symmetry [2] given by:

$$
Q\left(x, x_{1}, x_{2}, x_{12}, \alpha_{1}, \alpha_{2}\right)=Q\left(x_{1}, x, x_{12}, x_{2}, \alpha_{1}, \alpha_{2}\right) .
$$

Eq. (1.9) implies:

$$
Q=|Q, \quad \underline{Q}=| \underline{Q} .
$$

Geometrically the trapezoidal symmetry is an invariance with respect to the axis parallel to $\left(x_{1}, x_{12}\right)$. There might be a trapezoidal symmetry also with respect to the reflection around an axe parallel to $\left(x_{2}, x_{12}\right)$, but this can be reduced to the previous one by a rotation. So there is no need to treat such symmetry but it is sufficient to consider (1.10).

A detailed study of all the lattices derived from the rhombic $H^{4}$ family, including the construction of their three-leg forms, Lax pairs, Bäcklund transformations and infinite hierarchies of generalized symmetries, was presented in 31 .

A procedure for the embedding of the equations defined on a cell into a $3 \mathrm{D}$ consistent lattice is given in [8, 10. Different embeddings in $3 D$ consistent lattices resulting either in integrable or non integrable equations are discussed in 23 using the algebraic entropy analysis.

After the first results of Adler Bobenko and Suris [1] there have been various attempts to reduce the requirements imposed on consistent quad equations. Four non tetrahedral models, three of them with $D_{4}$ symmetry, were presented in 20,21. All of these models turn out to be more or less trivially linearizable [26]. Other consistent systems of quadrilateral lattice equations non possessing the tetrahedral property were studied in [2,5].

In the following we study the independent lattice equations (consistent on the cube) not already considered in the literature [1,2,31, i.e. those possessing the trapezoidal symmetry or with no symmetry at all. In Section 2 we list all independent equations defined on a cell and in Section 3 show in detail how one can extend the Möbius symmetry which classify Boll lattice equations defined on a four color lattice discussed in Appendix A. In Section 4 we present all the independent lattice equations obtained in this way and in Section 5 we analyze them from the point of view of the algebraic entropy showing that most of the new equations are linearizable. In Section 6 we explicitly linearize a few examples. Section 7 is devoted to some concluding remarks. 


\section{Independent equations on a single cell}

In Theorems 3.9 - 3.14 [10, Boll classified up to a $(\text { Möb })^{8}$ symmetry every consistent six-tuples of equations with the tetrahedron property. Here we consider all the independent quad equations defined on a single cell not of type $Q$ $\left(Q_{1}^{\varepsilon}, Q_{2}^{\varepsilon}, Q_{3}^{\varepsilon}\right.$ and $\left.Q_{4}\right)$ or rhombic $H^{4}\left({ }_{r} H_{1}^{\varepsilon},{ }_{r} H_{2}^{\varepsilon}\right.$ and $\left.{ }_{r} H_{3}^{\varepsilon}\right)$ as these two families have been already studied extensively [1,2,31. By independent we mean that the equations are defined up to a (Möb $)^{4}$ symmetry on the fields, rotations, translations and inversions of the reference system. By reference system we mean those two vectors applied on the point $x$ which define the two oriented directions $i$ and $j$ upon which the elementary square is constructed. The vertex of the square lying on direction $i(j)$ is then indicated by $x_{i}\left(x_{j}\right)$. The remaining vertex is then called $x_{i j}$. In Fig. 1 1 one can see an elementary square where $i=1$ and $j=2$ or viceversa.

The list we present in the following expands the analogous one given by Theorems 2.8-2.9 in [10], where the author does not distinguish between different arrangements of the fields $x_{i}, i=1, \ldots, 4$ over the four corners of the elementary square. Different choices reflects in different biquadratic's patterns and, for any system presented in Theorems 2.8-2.9 in [10, it is easy to see that a maximum of three different choices may arise up to rotations, translations and inversions.

Theorem 1 All the independent consistent quad equations not of type $Q$ or rhombic $H^{4}$ are given, up to (Möb) ${ }^{4}$ transformations of the fields $x, x_{i}, x_{j}$ and $x_{i j}$ and rotations, translations and inversions of the reference system, by nine different representatives, three of $H^{4}$-type and six of $H^{6}$-type. We list them with their quadruples of discriminants and we identify the six-tuple where the equation appears by the theorem number indicated in [10] in the form 3.a.b, where $\boldsymbol{b}$ is the order of the six-tuple into the theorem 3.a.

The trapezoidal equations of type $H^{4}$ are:

${ }_{t} H_{1}^{\varepsilon},\left(\varepsilon^{2}, \varepsilon^{2}, 0,0\right):$ Eq. B of 3.10.1.

$$
\left(x-x_{2}\right)\left(x_{3}-x_{23}\right)-\alpha_{2}\left(1+\varepsilon^{2} x_{3} x_{23}\right)=0 .
$$

${ }_{t} H_{2}^{\varepsilon},\left(1+4 \varepsilon x, 1+4 \varepsilon x_{2}, 1,1\right):$ Eq. B of 3.10.2.

$$
\begin{aligned}
\left(x-x_{2}\right)\left(x_{3}-x_{23}\right) & +\alpha_{2}\left(x+x_{2}+x_{3}+x_{23}\right)+ \\
& +\frac{\varepsilon \alpha_{2}}{2}\left(2 x_{3}+2 \alpha_{3}+\alpha_{2}\right)\left(2 x_{23}+2 \alpha_{3}+\alpha_{2}\right)+ \\
& +\left(\alpha_{2}+\alpha_{3}\right)^{2}-\alpha_{3}^{2}+\frac{\varepsilon \alpha_{2}^{3}}{2}=0 .
\end{aligned}
$$

${ }_{t} H_{3}^{\varepsilon},\left(x^{2}-4 \delta^{2} \varepsilon^{2}, x_{2}^{2}-4 \delta^{2} \varepsilon^{2}, x_{3}^{2}, x_{23}^{2}\right):$ Eq. B of 3.10.3.

$$
\begin{aligned}
e^{2 \alpha_{2}}\left(x x_{23}+x_{2} x_{3}\right) & -\left(x x_{3}+x_{2} x_{23}\right)- \\
& -e^{2 \alpha_{3}}\left(e^{4 \alpha_{2}}-1\right)\left(\delta^{2}+\frac{\varepsilon^{2} x_{3} x_{23}}{e^{4 \alpha_{3}+2 \alpha_{2}}}\right)=0 .
\end{aligned}
$$

The equations of type $H^{6}$ are: 
$D_{1},(0,0,0,0)$ : Eq. A of 3.12.1 and 3.13.1.

$$
x+x_{1}+x_{2}+x_{12}=0 .
$$

This equation is invariant under any exchange of the fields.

$$
\begin{aligned}
& { }_{1} D_{2},\left(\delta_{1}^{2},\left(\delta_{1} \delta_{2}+\delta_{1}-1\right)^{2}, 1,0\right): \text { Eq. A of 3.12.2. } \\
& \qquad \delta_{2} x+x_{1}+\left(1-\delta_{1}\right) x_{2}+x_{12}\left(x+\delta_{1} x_{2}\right)=0 .
\end{aligned}
$$

$D_{3},(4 x, 1,1,1)$ : Eq. A of 3.12.3.

$$
x+x_{1} x_{2}+x_{1} x_{12}+x_{2} x_{12}=0 .
$$

This equation is invariant under the exchange $x_{1} \leftrightarrow x_{2}$.

${ }_{1} D_{4},\left(x^{2}+4 \delta_{1} \delta_{2} \delta_{3}, x_{1}^{2}, x_{12}^{2}, x_{2}^{2}\right)$ : Eq. A of 3.12.4.

$$
x x_{12}+x_{1} x_{2}+\delta_{1} x_{1} x_{12}+\delta_{2} x_{2} x_{12}+\delta_{3}=0 .
$$

This equation is invariant under the simultaneous exchanges $x_{1} \leftrightarrow x_{2}$ and $\delta_{1} \leftrightarrow \delta_{2}$.

${ }_{2} D_{2},\left(\delta_{1}^{2}, 0,1\left(\delta_{1} \delta_{2}+\delta_{1}-1\right)^{2}\right):$ Eq. C of 3.13.2.

$$
\delta_{2} x+\left(1-\delta_{1}\right) x_{3}+x_{13}+x_{1}\left(x+\delta_{1} x_{3}-\delta_{1} \lambda\right)-\delta_{1} \delta_{2} \lambda=0 .
$$

${ }_{3} D_{2},\left(\delta_{1}^{2}, 0,\left(\delta_{1} \delta_{2}+\delta_{1}-1\right)^{2}, 1\right):$ Eq. C of 3.13.3.

$$
\delta_{2} x+x_{3}+\left(1-\delta_{1}\right) x_{13}+x_{1}\left(x+\delta_{1} x_{13}-\delta_{1} \lambda\right)-\delta_{1} \delta_{2} \lambda=0
$$

${ }_{2} D_{4},\left(x^{2}+4 \delta_{1} \delta_{2} \delta_{3}, x_{1}^{2}, x_{2}^{2}, x_{12}^{2}\right):$ Eq. A of 3.13.5.

$$
x x_{1}+\delta_{2} x_{1} x_{2}+\delta_{1} x_{1} x_{12}+x_{2} x_{12}+\delta_{3}=0 .
$$

This equation is invariant under the simultaneous exchanges $x_{2} \leftrightarrow x_{12}$ and $\delta_{1} \leftrightarrow \delta_{2}$

Let us note that differently from the rhombic $H^{4}$ equations, which are $\varepsilon$ deformations of the $H$ equations in the ABS classification [1] and hence, in the limit $\varepsilon \rightarrow 0$, have square symmetries, the trapezoidal $H^{4}$ equations in the limit $\varepsilon \rightarrow 0$ keep their discrete symmetry. Such class is then completely new with respect the ABS classification and the "deformed" and the "undeformed" equations share the same properties.

Everything is just written on a single cell and no dynamical system over the entire lattice exists. The problem of the embedding in a $2 D / 3 D$-lattice is discussed later in Appendix A. 


\section{Transformation groups for quad lattice equa- tions.}

We summarize in detail in Appendix A the results contained in [10] on the construction of the lattice equations and their Lax pairs.

Here in the following we present the extension of the Möbius transformations necessary to treat the equations we obtain when constructing quad graph equations consistent on an extended four color cube.

The classification of quad equations presented in Section 2 , has been carried out up to a Möbius transformations in each vertex:

$$
M:\left(x, x_{1}, x_{2}, x_{12}\right) \mapsto\left(\frac{a_{0} x+b_{0}}{c_{0} x+d_{0}}, \frac{a_{1} x_{1}+b_{1}}{c_{1} x_{1}+d_{1}}, \frac{a_{2} x_{2}+b_{2}}{c_{2} x_{2}+d_{2}}, \frac{a_{12} x_{12}+b_{12}}{c_{12} x_{12}+d_{12}}\right) .
$$

As in the usual Möbius transformation we have here $\left(a_{i}, b_{i}, c_{i}, d_{i}\right) \in \mathbb{C P}^{4} \backslash$ $V\left(a_{i} d_{i}-b_{i} c_{i}\right) \simeq \operatorname{PGL}(2, \mathbb{C})$ with $i=0,1,2,12$, i.e. each set of parameters is defined up to to a multiplication by a number. Obviously as the usual Möbius transformations these transformations will form a group under composition and we shall call such group (Möb) ${ }^{4}$.

On the other hand when dealing with equations defined on the lattice we have to follow the prescription of Appendix $\mathrm{A}$ and use the representation given by (A.14), i.e. we will have non-autonomous lattice equations. In this Section one proves the following Theorem which extends the group (Möb $)^{4}$ to the level of the transformations of the non autonomous lattice equations. We will call such group the "non-autonomous lifting" of (Möb) ${ }^{4}$, and denote it by $\widehat{(\mathrm{Möb})}^{4}$.

Theorem $2 \widehat{(\mathrm{Möb})}^{4}$ is the symmetry group of the non-autonomous difference equations obtained with the procedure presented in Appendix A by the "nonautonomous lifting" of (Möb $)^{4}$, the symmetry group of the quad equations presented in Section 2 .

This Theorem states that the result that we get by acting with a group of transformations $\widehat{(\mathrm{Möb})}^{4}$ is the same of what we obtain if we first transform

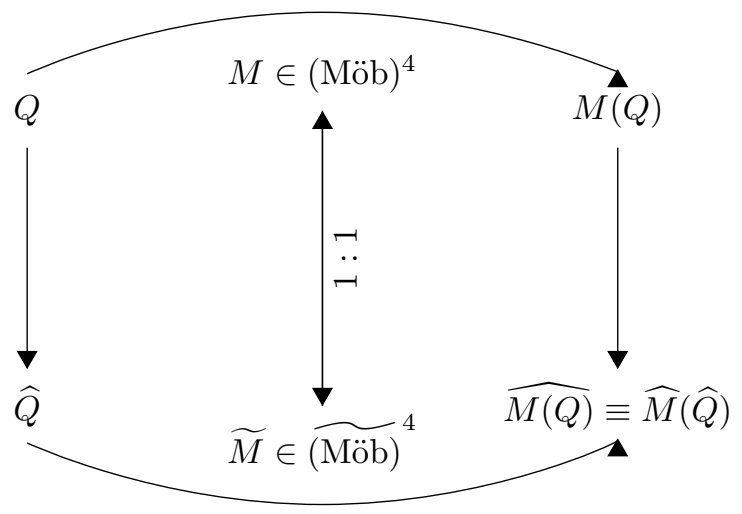

Figure 4: The commutative diagram defining $\widehat{(\mathrm{Möb})}^{4}$. 
the equations of Section 2 using $M \in(\mathrm{Möb})^{4}$ and then we construct the nonautonomous quad equation with the prescription of Appendix $\mathrm{A}$ or viceversa if we first construct the non-autonomous equation and then we transform it using the "non-autonomous lifing" of $M, \widehat{M}$, see Figure 4.

The Theorem is made up of two parts, the proof that $\widehat{(\mathrm{Möb})}^{4}$ is a group and that it is equivalent to the "non-autonomous lifting" of (Möb) ${ }^{4}$.

Let us first construct, a transformation which will be the candidate to be the "non-autonomous lifting" of $M \in(\mathrm{Möb})^{4}$. Given $M \in(\mathrm{Möb})^{4}$ using the same ideas of Appendix $\mathrm{A}$ we can construct the following transformation:

$$
\begin{aligned}
M_{n, m} \in \widehat{(\mathrm{Möb}}^{4}: u_{n, m} \mapsto & f_{n, m} \frac{a_{0} u_{n, m}+b_{0}}{c_{0} u_{n, m}+d_{0}}+\mid f_{n, m} \frac{a_{1} u_{n, m}+b_{1}}{c_{1} u_{n, m}+d_{1}}+ \\
& \underline{f}_{n, m} \frac{a_{2} u_{n, m}+b_{2}}{c_{2} u_{n, m}+d_{2}}+\mid \underline{f}_{n, m} \frac{a_{12} u_{n, m}+b_{12}}{c_{12} u_{n, m}+d_{12}} .
\end{aligned}
$$

Eq. (3.2) give us a mapping $\Phi$ between the group (Möb) ${ }^{4}$ and a set of nonautonomous transformations of the field $u_{n, m}$. Moreover there is a one to one correspondence between an element $M \in(\mathrm{Möb})^{4}$ (3.1) and one $M_{n, m} \in \widehat{(\mathrm{Möb})}^{4}$ (3.2). $\Phi$ is given by:

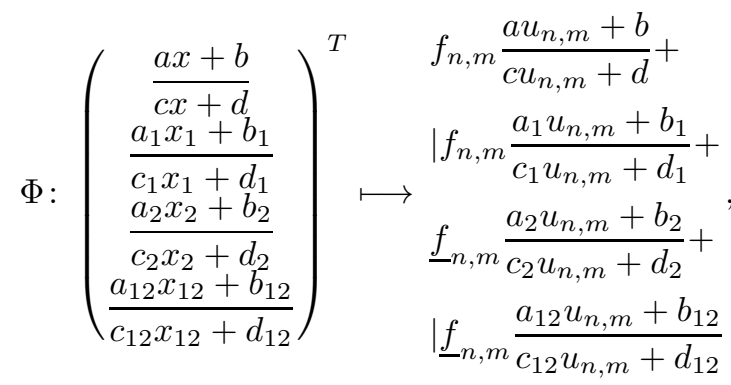

$$
\begin{aligned}
& \begin{array}{c}
f_{n, m} \frac{\alpha^{(0)} u_{n, m}+\beta^{(0)}}{\gamma^{(0)} u_{n, m}+\delta^{(0)}}+ \\
\mid f_{n, m} \frac{\alpha^{(1)} u_{n, m}+\beta^{(1)}}{\gamma^{(1)} u_{n, m}+\delta^{(1)}}+ \\
\left.\Phi^{-1}: \begin{array}{c}
\frac{\alpha^{(0)} x+\beta^{(0)}}{\gamma^{(0)} x+\delta^{(0)}} \\
\frac{\alpha^{(1)} x_{1}+\beta^{(1)}}{\gamma^{(1)} x_{1}+\delta^{(1)}} \\
\frac{\alpha^{(2)} x_{2}+\beta^{(2)}}{\gamma_{n, m}^{(2)} x_{2}+\delta^{(2)}} \\
\quad \frac{\alpha^{(2)} u_{n, m}+\beta^{(2)}}{\gamma^{(2)} u_{n, m}+\delta^{(2)}}+ \\
\quad \underline{f}_{n, m} \frac{\alpha^{(3)} x_{12}+\beta^{(3)}}{\gamma^{(3)} u_{n, m}+\beta^{(3)}}
\end{array}\right)^{T(3)} x_{12}+\delta^{(3)}
\end{array}
\end{aligned}
$$

We have now to prove that $\widehat{(M o ̈ b)}^{4}$ is a group and that the mapping (3.3) is actually a group homomorphism.

$\widehat{(\mathrm{Möb})}^{4}$ is a subset of the general non-autonomous Möbius transformation:

$$
W_{n, m}: u_{n, m} \mapsto \frac{a_{n, m} u_{n, m}+b_{n, m}}{c_{n, m} u_{n, m}+d_{n, m}} .
$$

From the general rule of composition of two Möbius transformations (3.4) we 
get:

$$
W_{n, m}^{1}\left(W_{n, m}^{0}\left(u_{n, m}\right)\right)=\frac{\left(a_{n, m}^{0} a_{n, m}^{1}+b_{n, m}^{1} c_{n, m}^{0}\right) u_{n, m}+a_{n, m}^{1} b_{n, m}^{0}+b_{n, m}^{1} d_{n, m}^{0}}{\left(a_{n, m}^{0} c_{n, m}^{1}+c_{n, m}^{0} d_{n, m}^{1}\right) u_{n, m}+b_{n, m}^{0} c_{n, m}^{1}+d_{n, m}^{0} d_{n, m}^{1}} .
$$

Its inverse is given by

$$
W_{n, m}^{-1}\left(u_{n, m}\right)=\frac{d_{n, m} u_{n, m}-b_{n, m}}{-c_{n, m} u_{n, m}+a_{n, m}}
$$

Using the computational rules given in Table 1 and in (3.5) we find that the composition of two elements $M_{n, m}^{(1)}, M_{n, m}^{(2)} \in \widehat{(\mathrm{Möb})}^{4}$ with parameters $\left(a_{j}^{(i)}, b_{j}^{(i)}, c_{j}^{(i)}, d_{j}^{(i)}\right)$, $j=0,1,2,3$ and $i=1,2$, gives:

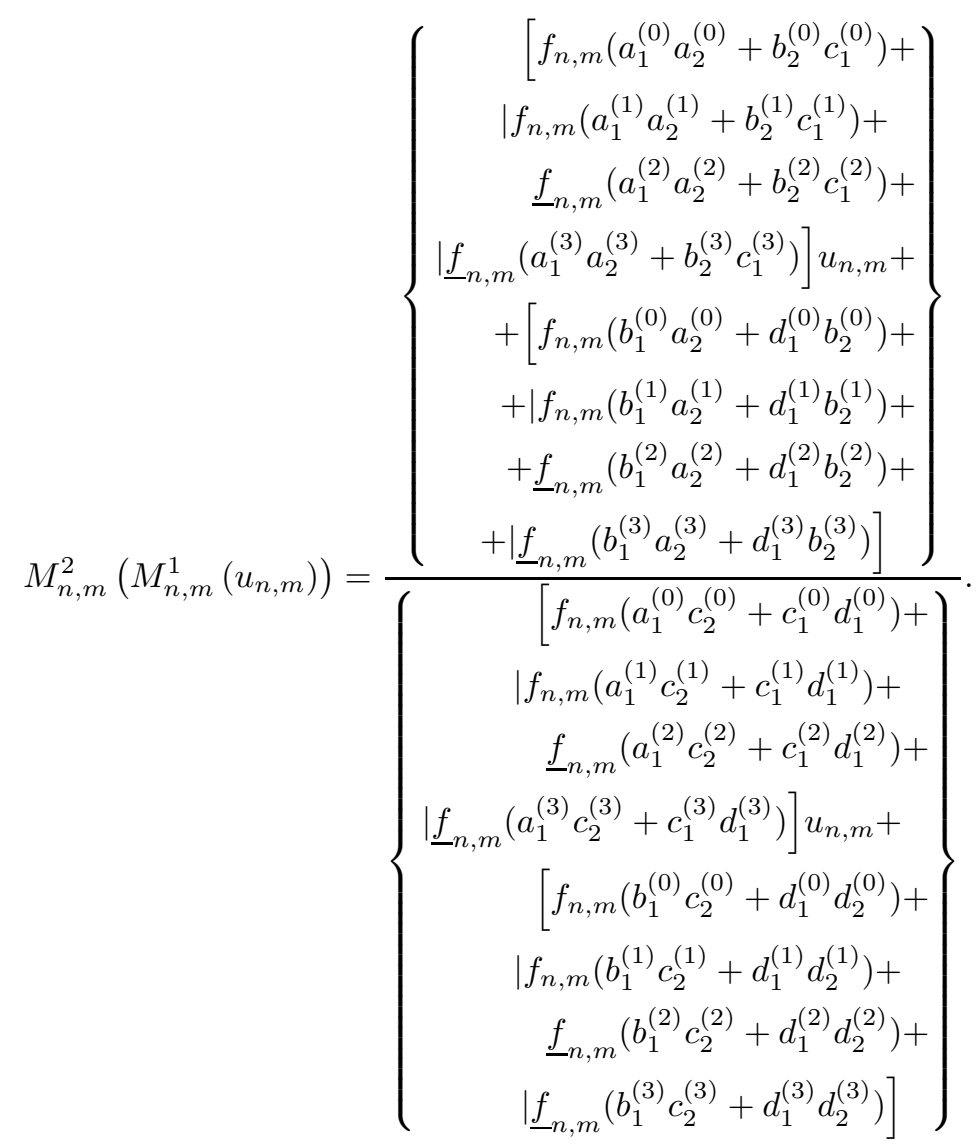

Its inverse is given by

$$
M_{n, m}^{-1}\left(u_{n, m}\right)=\frac{\left\{\begin{array}{l}
\left(f_{n, m} d^{(0)}+\left|f_{n, m} d_{1}+\underline{f}_{n, m} d_{2}+\right| \underline{f}_{n, m} d_{3}\right) u_{n, m}- \\
\left(f_{n, m} b_{0}+\left|f_{n, m} b_{1}+\underline{f}_{n, m} b_{2}+\right| \underline{f}_{n, m} b_{3}\right)
\end{array}\right\}}{\left\{\begin{array}{c}
-\left(f_{n, m} c_{0}+\left|f_{n, m} c_{1}+\underline{f}_{n, m} c_{2}+\right| \underline{f}_{n, m} c_{3}\right) u_{n, m}+ \\
\left(f_{n, m} a_{0}+\left|f_{n, m} a_{1}+\underline{f}_{n, m} a_{2}+\right| \underline{f}_{n, m} a_{3}\right)
\end{array}\right\}} .
$$

Thus one has proven that $\widehat{(\mathrm{Möb})}^{4}$ is a group. 


\begin{tabular}{ccccc}
\hline & $f_{n, m}$ & $\mid f_{n, m}$ & $\underline{f}_{n, m}$ & $\mid \underline{f}_{n, m}$ \\
\hline$f_{n, m}$ & $f_{n, m}$ & 0 & 0 & 0 \\
$\mid f_{n, m}$ & 0 & $\mid f_{n, m}$ & 0 & 0 \\
$\underline{f}_{n, m}$ & 0 & 0 & $\underline{f}_{n, m}$ & 0 \\
$\underline{f}_{n, m}$ & 0 & 0 & 0 & $\mid \underline{f}_{n, m}$ \\
\hline
\end{tabular}

Table 1: Multiplication rules for the functions $\tilde{f}_{n, m}$ as given by (A.18).

Let us show that the maps $\Phi$ and $\Phi^{-1}$ in (3.3) are group homomorphism. They preserve the identity, and from the formula of composition of Möbius transformations in (Möb) ${ }^{4}$ (3.7) we derive the required result.

Let us now check if the diagram of Fig. 4 is satisfied. Let us consider (3.1) and (3.2) and a general multilinear quad-equation:

$$
\begin{aligned}
Q_{\text {gen }}\left(x, x_{1}, x_{2}, x_{12}\right) & =A_{0,1,2,12} x x_{1} x_{2} x_{12}+B_{0,1,2} x x_{1} x_{2}+ \\
& +B_{0,1,12} x x_{1} x_{12}+B_{0,2,12} x x_{2} x_{12}+B_{1,2,12} x_{1} x_{2} x_{12} \\
& +C_{0,1} x x_{1}+C_{0,2} x x_{2}+C_{0,12} x x_{12}+C_{1,2} x_{1} x_{2} \\
& +C_{1,12} x_{1} x_{12}+C_{2,12} x_{2} x_{12} \\
& +D_{0} x+D_{1} x_{1}+D_{2} x_{2}+D_{12} x_{12}+K
\end{aligned}
$$

where $A_{0,1,2,12}, B_{i, j, k}, C_{i, j}, D_{i}$ and $K$ with $i, j, k \in\{0,1,2,12\}$ are arbitrary complex constants. The proof that $Q\left(M\left(x, x_{1}, x_{2}, x_{12}\right)\right)=\widehat{Q}\left(M_{n, m}\left(u_{n, m}\right)\right)$ where $M \in(\mathrm{Möb})^{4}$ and $M_{n, m}=\Phi(M) \in \widehat{(\mathrm{Möb})}^{4}$ is a very computationally heavy calculation due to the high number of parameters involved (twelve in the transformation 2 and fifteen in the equation (3.9), twenty-seven paramrters in total) and to the fact that rational functions are involved. To simplify the problem it is sufficient to recall that every Möbius

$$
m(z)=\frac{a z+b}{c z+d}, \quad z \in \mathbb{C}
$$

transformation can be obtained as a superposition of a translation:

$$
T_{a}(z)=z+a,
$$

dilatation:

$$
D_{a}(z)=a z
$$

and inversion:

$$
I(z)=\frac{1}{z}
$$

i.e.

$$
m(z)=\left(T_{a / c} \circ D_{(b c-a d) / c^{2}} \circ I \circ T_{d / c}\right)(z) .
$$

\footnotetext{
${ }^{2}$ Using that Möbius transformations are projectively defined one can lower the number of parameters from sixteen to twelve, but this implies to impose that some parameters are non-zero and thus all various different possibilities must be taken into account.
} 
As the group (Möb) ${ }^{4}$ is obtained by four copies of the Möbius group each acting on a different variable we can decompose each entry $M \in(\mathrm{Möb})^{4}$ as in (3.12). Therefore we need to check $3^{4}=81$ transformations, depending at most on four parameters. We can automatize such proof by making a specific computer program to generate all the possible fundamental transformations in (Möb $)^{4}$ and then check them one by one reducing the computational effort. To this end we used the Computer Algebra System (CAS) SymPy [27. This ends the proof of the Theorem. The details of the calculations will be contained in [16].

\section{Independent equations on the $2 D$-lattice}

We will now extend to the $2 D$-lattice all the systems listed in Theorem 1 of Section 2 according to the prescription given in Appendix A Independence is now understood to be up to $\widehat{(\mathrm{Möb})} 4$ symmetry and rotations, translations and inversions of the reference system. The transformations of the reference system are take to be acting on the discrete indexes rather than on the reference frame. For sake of compactness and as the equations are on the lattice we shall omit the hats on the Möbius transformations when clear.

Theorem 3 All the independent nonlinear, 2D-dynamical systems not of type $Q$ or rhombic $H^{4}$ which are consistent on the $3 D$-lattice are given, up to $\widehat{(\mathrm{Möb})} 4$ transformations of the fields $u_{n, m}, u_{n, m+1}, u_{n+1, m}$ and $u_{n+1, m+1}$, rotations, translations and inversions of the discrete indexes $n$ and $m$, by nine non autonomous representatives, three of trapezoidal $H^{4}$-type and six of $H^{6}$-type.

The $H^{4}$ type equations are:

$$
\begin{aligned}
{ }_{t} H_{1}: & \left(u_{n, m}-u_{n+1, m}\right) \cdot\left(u_{n, m+1}-u_{n+1, m+1}\right)- \\
& -\alpha_{2} \varepsilon^{2}\left(F_{m}^{(+)} u_{n, m+1} u_{n+1, m+1}+F_{m}^{(-)} u_{n, m} u_{n+1, m}\right)-\alpha_{2}=0, \\
{ }_{t} H_{2}: & \left(u_{n, m}-u_{n+1, m}\right)\left(u_{n, m+1}-u_{n+1, m+1}\right) \\
& +\alpha_{2}\left(u_{n, m}+u_{n+1, m}+u_{n, m+1}+u_{n+1, m+1}\right) \\
& +\frac{\varepsilon \alpha_{2}}{2}\left(2 F_{m}^{(+)} u_{n, m+1}+2 \alpha_{3}+\alpha_{2}\right)\left(2 F_{m}^{(+)} u_{n+1, m+1}+2 \alpha_{3}+\alpha_{2}\right) \\
& +\frac{\varepsilon \alpha_{2}}{2}\left(2 F_{m}^{(-)} u_{n, m}+2 \alpha_{3}+\alpha_{2}\right)\left(2 F_{m}^{(-)} u_{n+1, m}+2 \alpha_{3}+\alpha_{2}\right) \\
& +\left(\alpha_{3}+\alpha_{2}\right)^{2}-\alpha_{3}^{2}-2 \varepsilon \alpha_{2} \alpha_{3}\left(\alpha_{3}+\alpha_{2}\right)=0 \\
{ }_{t} H_{3}: & \alpha_{2}\left(u_{n, m} u_{n+1, m+1}+u_{n+1, m} u_{n, m+1}\right) \\
& -\left(u_{n, m} u_{n, m+1}+u_{n+1, m} u_{n+1, m+1}\right)-\alpha_{3}\left(\alpha_{2}^{2}-1\right) \delta^{2}+ \\
& -\frac{\varepsilon^{2}\left(\alpha_{2}^{2}-1\right)}{\alpha_{3} \alpha_{2}}\left(F_{m}^{(+)} u_{n, m+1} u_{n+1, m+1}+F_{m}^{(-)} u_{n, m} u_{n+1, m}\right)=0,
\end{aligned}
$$

These equations arise from the $B$ equation of the cases 3.10.1, 3.10.2 and 3.10.3 in [10 respectively.

The $H^{6}$ type equations are:

$$
D_{1}: u_{n, m}+u_{n+1, m}+u_{n, m+1}+u_{n+1, m+1}=0 \text {. }
$$


${ }_{1} D_{2}: \quad\left(F_{n+m}^{(-)}-\delta_{1} F_{n}^{(+)} F_{m}^{(-)}+\delta_{2} F_{n}^{(+)} F_{m}^{(+)}\right) u_{n, m}$

$+\left(F_{n+m}^{(+)}-\delta_{1} F_{n}^{(-)} F_{m}^{(-)}+\delta_{2} F_{n}^{(-)} F_{m}^{(+)}\right) u_{n+1, m}+$

$+\left(F_{n+m}^{(+)}-\delta_{1} F_{n}^{(+)} F_{m}^{(+)}+\delta_{2} F_{n}^{(+)} F_{m}^{(-)}\right) u_{n, m+1}$

$+\left(F_{n+m}^{(-)}-\delta_{1} F_{n}^{(-)} F_{m}^{(+)}+\delta_{2} F_{n}^{(-)} F_{m}^{(-)}\right) u_{n+1, m+1}+$

$+\delta_{1}\left(F_{m}^{(-)} u_{n, m} u_{n+1, m}+F_{m}^{(+)} u_{n, m+1} u_{n+1, m+1}\right)$

$+F_{n+m}^{(+)} u_{n, m} u_{n+1, m+1}+F_{n+m}^{(-)} u_{n+1, m} u_{n, m+1}=0$,

${ }_{2} D_{2}: \quad\left(F_{m}^{(-)}-\delta_{1} F_{n}^{(+)} F_{m}^{(-)}+\delta_{2} F_{n}^{(+)} F_{m}^{(+)}-\delta_{1} \lambda F_{n}^{(-)} F_{m}^{(+)}\right) u_{n, m}$

$+\left(F_{m}^{(-)}-\delta_{1} F_{n}^{(-)} F_{m}^{(-)}+\delta_{2} F_{n}^{(-)} F_{m}^{(+)}-\delta_{1} \lambda F_{n}^{(+)} F_{m}^{(+)}\right) u_{n+1, m}$

$+\left(F_{m}^{(+)}-\delta_{1} F_{n}^{(+)} F_{m}^{(+)}+\delta_{2} F_{n}^{(+)} F_{m}^{(-)}-\delta_{1} \lambda F_{n}^{(-)} F_{m}^{(-)}\right) u_{n, m+1}$

$+\left(F_{m}^{(+)}-\delta_{1} F_{n}^{(-)} F_{m}^{(+)}+\delta_{2} F_{n}^{(-)} F_{m}^{(-)}-\delta_{1} \lambda F_{n}^{(+)} F_{m}^{(-)}\right) u_{n+1, m+1}$

$+\delta_{1}\left(F_{n+m}^{(-)} u_{n, m} u_{n+1, m+1}+F_{n+m}^{(+)} u_{n+1, m} u_{n, m+1}\right)$

$+F_{m}^{(+)} u_{n, m} u_{n+1, m}+F_{m}^{(-)} u_{n, m+1} u_{n+1, m+1}-\delta_{1} \delta_{2} \lambda=0$,

${ }_{3} D_{2}: \quad\left(F_{m}^{(-)}-\delta_{1} F_{n}^{(-)} F_{m}^{(-)}+\delta_{2} F_{n}^{(+)} F_{m}^{(+)}-\delta_{1} \lambda F_{n}^{(-)} F_{m}^{(+)}\right) u_{n, m}$

$+\left(F_{m}^{(-)}-\delta_{1} F_{n}^{(+)} F_{m}^{(-)}+\delta_{2} F_{n}^{(-)} F_{m}^{(+)}-\delta_{1} \lambda F_{n}^{(+)} F_{m}^{(+)}\right) u_{n+1, m}$

$+\left(F_{m}^{(+)}-\delta_{1} F_{n}^{(-)} F_{m}^{(+)}+\delta_{2} F_{n}^{(+)} F_{m}^{(-)}-\delta_{1} \lambda F_{n}^{(-)} F_{m}^{(-)}\right) u_{n, m+1}$

$+\left(F_{m}^{(+)}-\delta_{1} F_{n}^{(+)} F_{m}^{(+)}+\delta_{2} F_{n}^{(-)} F_{m}^{(-)}-\delta_{1} \lambda F_{n}^{(+)} F_{m}^{(-)}\right) u_{n+1, m+1}$

$+\delta_{1}\left(F_{n}^{(-)} u_{n, m} u_{n, m+1}+F_{n}^{(+)} u_{n+1, m} u_{n+1, m+1}\right)$

$+F_{m}^{(-)} u_{n, m+1} u_{n+1, m+1}+F_{m}^{(+)} u_{n, m} u_{n+1, m}-\delta_{1} \delta_{2} \lambda=0$,

$D_{3}: \quad F_{n}^{(+)} F_{m}^{(+)} u_{n, m}+F_{n}^{(-)} F_{m}^{(+)} u_{n+1, m}+F_{n}^{(+)} F_{m}^{(-)} u_{n, m+1}$

$+F_{n}^{(-)} F_{m}^{(-)} u_{n+1, m+1}+F_{m}^{(-)} u_{n, m} u_{n+1, m}$

$+F_{n}^{(-)} u_{n, m} u_{n, m+1}+F_{n+m}^{(-)} u_{n, m} u_{n+1, m+1}+$

$+F_{n+m}^{(+)} u_{n+1, m} u_{n, m+1}+F_{n}^{(+)} u_{n+1, m} u_{n+1, m+1}$

$+F_{m}^{(+)} u_{n, m+1} u_{n+1, m+1}=0$,

${ }_{1} D_{4}: \quad \delta_{1}\left(F_{n}^{(-)} u_{n, m} u_{n, m+1}+F_{n}^{(+)} u_{n+1, m} u_{n+1, m+1}\right)+$

$+\delta_{2}\left(F_{m}^{(-)} u_{n, m} u_{n+1, m}+F_{m}^{(+)} u_{n, m+1} u_{n+1, m+1}\right)+$

$+u_{n, m} u_{n+1, m+1}+u_{n+1, m} u_{n, m+1}+\delta_{3}=0$,

${ }_{2} D_{4}: \quad \delta_{1}\left(F_{n}^{(-)} u_{n, m} u_{n, m+1}+F_{n}^{(+)} u_{n+1, m} u_{n+1, m+1}\right)+$

$+\delta_{2}\left(F_{n+m}^{(-)} u_{n, m} u_{n+1, m+1}+F_{n+m}^{(+)} u_{n+1, m} u_{n, m+1}\right)+$

$+u_{n, m} u_{n+1, m}+u_{n, m+1} u_{n+1, m+1}+\delta_{3}=0$.

The equations ${ }_{1} D_{2},{ }_{1} D_{4},{ }_{2} D_{2}$ and $D_{3}$ arise from the $A$ equation in the cases 
3.12.2, 3.12.3, 3.12.4 and 3.13.5 respectively. Instead equations ${ }_{2} D_{2}$ and ${ }_{3} D_{2}$ arise from the $C$ equation in the cases 3.13.2 and 3.13.3 respectively.

To write down the explicit form of the equations in (4.2) we used A.20. A.23) and the fact that the following identities holds:

$$
\begin{aligned}
f_{n, m}=F_{n}^{(+)} F_{m}^{(+)}, & \mid f_{n, m}=F_{n}^{(-)} F_{m}^{(+)}, \\
\underline{f}_{n, m}=F_{n}^{(+)} F_{m}^{(-)}, & \mid \underline{f}_{n, m}=F_{n}^{(-)} F_{m}^{(-)} .
\end{aligned}
$$

As mentioned in Appendix $\mathrm{A}$ if we apply this procedure to an equation of rhombic type we get a result consistent with [31.

\section{Algebraic Entropy test for (4.1, 4.2)}

Algebraic Entropy is used as a test of integrability for discrete systems. It measures the degree of growth of the iterates of a rational map [28, 29]. The classification of lattice equations based on the algebraic entropy test $[22]$ is:

Linear growth: The equation is linearizable.

Polynomial growth: The equation is integrable.

Exponential growth: The equation is chaotic.

We have performed the Algebraic Entropy analysis in the principal growth directions 29 as shown in Figure 5 on all non-autonomous equations presented in Section 4 to identify their behaviour. To this end we used the SymPy [27] module ae2d.py [17]. We found that a non-autonomous equation, at difference from what it is assumed in [29, may have non-constant degrees upon the diagonals. This fact implies that the equations of Section 4 do not have in general a single sequence of degrees.

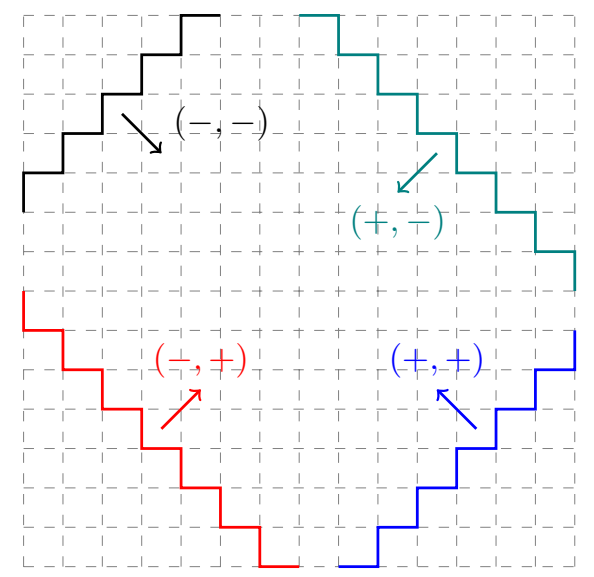

Figure 5: Principal growth directions.

Let us explain this result in details. Let us suppose that we wish to calculate the Algebraic Entropy in the North-East direction $(-,+)$. We should then solve 
our equation with respect to $u_{n+1, m+1}$ and then iteratively find the degree of the map in the $(-,+)$ direction. According to 29 one obtains a matrix, the evolution matrix, where in each element we put the degree $d_{k}$ of the corresponding $k$ lattice point. In the evolution matrix the degrees along the diagonals are assumed to be all equal. Then the evolution matrix will have the form:

$$
\begin{array}{cccccc}
1 & d_{2} & d_{3} & d_{4} & d_{5} & d_{6} \\
1 & 1 & d_{2} & d_{3} & d_{4} & d_{5} \\
& 1 & 1 & d_{2} & d_{3} & d_{4} \\
& & 1 & 1 & d_{2} & d_{3} \\
& & & 1 & 1 & d_{2} \\
& & & & 1 & 1
\end{array}
$$

The Algebraic Entropy $\eta$ is defined as:

$$
\eta=\lim _{k \rightarrow \infty} \frac{1}{k} \log d_{k}
$$

In our case we obtain experimentally that the degrees along the diagonals are not the same. This means that the actual evolution matrix will have the form:

$$
\begin{array}{cccccc}
1 & d_{2}^{(5)} & d_{3}^{(4)} & d_{4}^{(3)} & d_{5}^{(2)} & d_{6}^{(1)} \\
1 & 1 & d_{2}^{(4)} & d_{3}^{(3)} & d_{4}^{(2)} & d_{5}^{(1)} \\
& 1 & 1 & d_{2}^{(3)} & d_{3}^{(2)} & d_{4}^{(1)} \\
& & 1 & 1 & d_{2}^{(2)} & d_{3}^{(1)} \\
& & & 1 & 1 & d_{2}^{(1)} \\
& & & & 1 & 1
\end{array}
$$

where by $d_{k}^{(i)}$ we mean the degree of the iterate map proper to the $i$-th column of the evolution matrix. This is obviously a more general case than (5.1). In the framework of [29] the sequence of degrees will have the form (5.1) but in our case we get (5.3). We shall call the sequence

$$
1, d_{2}^{(1)}, d_{3}^{(1)}, d_{4}^{(1)}, d_{5}^{(1)}, \ldots
$$

the principal sequence of growth. A sequence as

$$
1, d_{2}^{(i)}, d_{3}^{(i)}, d_{4}^{(i)}, d_{5}^{(i)}, \ldots
$$

with $i=2$ will be a secondary sequence of growth, for $i=3$ a third, and so on. In principle any sequence of the form (5.5) will define an entropy $\eta^{(i)}$ :

$$
\eta^{(i)}=\lim _{k \rightarrow \infty} \frac{1}{k} \log d_{k}^{(i)} .
$$

To any sequence of the form (5.5) we can associate a generating function, i.e. the function $g^{(i)}(s)$ such that the coefficients of its Taylor expansion care as near as possible to the $d_{k}^{(i)}$ :

$$
g^{(i)}(s)=\sum_{k=0}^{\infty} d_{k}^{(i)} s^{i} .
$$


Usually we assume these generating functions to be rational and then the Algebraic Entropy can be calculated as the modulus of the smallest pole of the generating function:

$$
\eta^{(i)}=\min \left\{|s|\left|\lim _{\sigma \rightarrow s}\right| g^{(i)}(\sigma) \mid=\infty\right\} .
$$

We built the program ae2d.py to analyze the evolution matrices and to search for recurring sequences of growth. It can extract from a (sufficiently big) evolution matrix the number of sequences of growth and analyze them. For further details see [16, 17.

Before considering the Algebraic Entropy of the equations presented in Section 4 we discuss briefly the Algebraic Entropy for the rhombic $H^{4}$ equations, which are well known to be integrable [31. Running ae2d.py on these equations one finds that they possess only a principal sequence with the following isotropic sequence of degrees:

$$
1,2,4,7,11,16,22,29,37, \ldots
$$

To this sequence corresponds the generating function:

$$
g=\frac{s^{2}-s+1}{(1-s)^{3}}
$$

which gives, through the definition $g=\sum_{k} d_{k} s^{k}$, the asymptotic fit of the degrees:

$$
d_{k}=\frac{k(k+1)}{2}+1 .
$$

Since the growth is quadratic $\eta=0$. This result is a confirmation by the Algebraic Entropy approach of the integrability of the rhombic $H^{4}$ equations. Let us note that the growth sequences (5.9) are the same in the Rhombic $H^{4}$ equations also when $\varepsilon=0$, i.e. if we are in the case of the $H$ equations of the ABS classification.

For the trapezoidal $H^{4}$ and $H^{6}$ equations the situation is a bit more complicate. Indeed those equations have in every direction two different sequence of growth, the principal and the secondary one, as the coefficients of the evolution matrix (5.3) are 2-periodic. The most surprising feature is however that all the sequences of growth are linear. This means that all such equations are not only integrable due to the $\mathrm{CAC}$ property, but also linearizable.

Instead of presenting the full evolution matrices (5.3), which would be very lengthily and obscure, we present two tables with the relevant properties. The interested reader will find the full matrices in [16. In Table2 we present a summary of the sequences of growth of both trapezoidal $H^{4}$ and $H^{6}$ equations. The explicit sequence of the degrees of growth with generating functions, asymptotic fit of the degrees of growth and entropy is given in Table 3 .

Observing Table 2 and Table 3 we may notice the following facts:

- The trapezoidal $H^{4}$ equations are not isotropic: the sequences in the $(-,+)$ and $(+,+)$ directions are different from those in the $(+,-)$ and $(-,-)$ directions. These results reflect the symmetry of the equations. 


\begin{tabular}{ccccc}
\hline Equation & \multicolumn{4}{c}{ Growth direction } \\
&,-+ &,++ &,+- &,-- \\
\hline${ }_{t} H_{1}^{\varepsilon}$ & $L_{1}, L_{2}$ & $L_{1}, L_{2}$ & $L_{3}, L_{4}$ & $L_{3}, L_{4}$ \\
${ }_{t} H_{2}^{\varepsilon}$ & $L_{5}, L_{6}$ & $L_{5}, L_{6}$ & $L_{7}, L_{8}$ & $L_{7}, L_{8}$ \\
${ }_{t} H_{3}^{\varepsilon}$ & $L_{5}, L_{6}$ & $L_{5}, L_{6}$ & $L_{7}, L_{8}$ & $L_{7}, L_{8}$ \\
$D_{1}$ & $L_{0}, L_{0}$ & $L_{0}, L_{0}$ & $L_{0}, L_{0}$ & $L_{0}, L_{0}$ \\
${ }_{1} D_{2}$ & $L_{9}, L_{10}$ & $L_{9}, L_{10}$ & $L_{9}, L_{10}$ & $L_{9}, L_{10}$ \\
$D_{3}$ & $L_{11}, L_{12}$ & $L_{11}, L_{12}$ & $L_{11}, L_{12}$ & $L_{11}, L_{12}$ \\
${ }_{1} D_{4}$ & $L_{13}, L_{8}$ & $L_{13}, L_{8}$ & $L_{13}, L_{8}$ & $L_{13}, L_{8}$ \\
${ }_{2} D_{2}$ & $L_{14}, L_{15}$ & $L_{14}, L_{15}$ & $L_{15}, L_{14}$ & $L_{15}, L_{14}$ \\
${ }_{3} D_{2}$ & $L_{16}, L_{17}$ & $L_{16}, L_{17}$ & $L_{17}, L_{16}$ & $L_{17}, L_{16}$ \\
${ }_{2} D_{4}$ & $L_{18}, L_{8}$ & $L_{18}, L_{8}$ & $L_{19}, L_{20}$ & $L_{19}, L_{20}$ \\
\hline
\end{tabular}

Table 2: Sequences of growth for the trapezoidal $H^{4}$ and $H^{6}$ equations. The first one is the principal sequence, while the second the secondary. All sequences $L_{j}, j=0, \cdots, 20$ are presented in Table 3 .

- The $H^{6}$ equations, except from ${ }_{2} D_{4}$ which has the same behaviour as the trapezoidal $H^{4}$, are isotropic. Equations ${ }_{2} D_{2}$ and ${ }_{3} D_{2}$ exchange the principal and the secondary sequences from the $(-,+),(+,+)$ directions and the $(+,-),(-,-)$ directions.

- All growths, except $L_{0}, L_{3}, L_{4}, L_{7}, L_{8}, L_{12}$ and $L_{17}$, exhibit a highly oscillatory behaviour. They have generating functions of the form:

$$
g(s)=\frac{P(s)}{(s-1)^{2}(s+1)^{2}},
$$

with the polynomial $P(s) \in \mathbb{Z}[s]$. We may write

$$
g(s)=P_{0}(s)+\frac{P_{1}(s)}{(s-1)^{2}(s+1)^{2}},
$$

with $P_{0}(s) \in \mathbb{Z}[s]$ of degree less than $P$ and $P_{1}(s)=\alpha s^{3}+\beta s^{2}+\gamma s+\delta$. Expanding the second term in (5.13) in partial fractions we obtain:

$$
\begin{aligned}
& g(s)=P_{0}(s)+\frac{1}{4}\left[\frac{-\alpha-\gamma+\beta+\delta}{(s+1)^{2}}+\frac{\alpha+\gamma+\beta+\delta}{(s-1)^{2}}\right. \\
& \left.+\frac{2 \alpha+\beta-\delta}{s-1}+\frac{2 \alpha-\beta+\delta}{s+1}\right] \text {. }
\end{aligned}
$$

Expanding the term in square parentheses in Taylor series we find that:

$$
g(s)=P_{0}(s)+\sum_{k=0}^{\infty}\left[A_{0}+A_{1}(-1)^{k}+A_{2} k+A_{3}(-1)^{k} k\right] s^{k},
$$

with $A_{i}=A_{i}(\alpha, \beta, \gamma, \delta)$ constants. This means the $d_{k}=A_{0}+A_{1}(-1)^{k}+$ $A_{2} k+A_{3}(-1)^{k} k$ for $k>\operatorname{deg} P_{0}(s)$, and therefore it asymptotically solves a 
fourth order difference equation. As far as we know, even if some example of behaviour containing terms like $(-1)^{k}$ are known [22, this is the first time that we observe patterns with oscillations given by $k(-1)^{k}$. We conclude noting that the usage of the algebraic entropy as integrability indicator is actually justified by the existence of of finite order recurrence relations between the degrees $d_{k}$. Indeed the existence of such recurrence relations means that from a local property (the sequence of degrees) we may infer a global one (chaoticity/integrability/linearizaribilty) 30. 
Table 3: Sequences of growth, generating functions, analytic expression of the degrees and entropy for the trapezoidal $H^{4}$ and $H^{6}$ equations

\begin{tabular}{|c|c|c|c|c|c|}
\hline \multirow{10}{*}{$\vec{\bullet}$} & Name & $\begin{array}{l}\text { Degrees } \\
\quad\left\{d_{k}\right\}\end{array}$ & $\begin{array}{l}\text { Generating function } \\
\qquad g(s)\end{array}$ & $\begin{array}{l}\text { Degree fit } \\
\quad d_{k}\end{array}$ & $\begin{array}{c}\text { Entropy } \\
\eta\end{array}$ \\
\hline & $L_{0}$ & $1,1,1,1,1,1,1,1,1,1,1,1,1 \ldots$ & $\frac{1}{1-s}$ & 1 & 0 \\
\hline & $L_{1}$ & $1,2,2,5,3,8,4,11,5,14,6,17,7 \ldots$ & $\frac{s^{3}+2 s+1}{(s-1)^{2}(s+1)^{2}}$ & $\frac{(-1)^{k}}{4}(-2 k+1)+k+\frac{3}{4}$ & 0 \\
\hline & $L_{2}$ & $1,2,4,3,7,4,10,5,13,6,16,7,19 \ldots$ & $\frac{-s^{3}+2 s^{2}+2 s+1}{(s-1)^{2}(s+1)^{2}}$ & $\frac{(-1)^{k}}{4}(2 k-1)+k+\frac{5}{4}$ & 0 \\
\hline & $L_{3}$ & $1,2,2,3,3,4,4,5,5,6,6,7,7 \ldots$ & $\frac{-s^{2}+s+1}{s^{3}-s^{2}-s+1}$ & $-\frac{(-1)^{k}}{4}+\frac{k}{2}+\frac{5}{4}$ & 0 \\
\hline & $L_{4}$ & $1,2,4,5,7,8,10,11,13,14,16,17,19 \ldots$ & $\frac{s^{2}+s+1}{s^{3}-s^{2}-s+1}$ & $\frac{(-1)^{k}}{4}+\frac{3 k}{2}+\frac{3}{4}$ & 0 \\
\hline & $L_{5}$ & $1,2,4,6,11,10,19,14,27,18,35,22,43 \ldots$ & $\frac{s^{6}+4 s^{4}+2 s^{3}+2 s^{2}+2 s+1}{(s-1)^{2}(s+1)^{2}}$ & $(-1)^{k}\left(k-\frac{5}{2}\right)^{2}+3 k-\frac{5}{2}$ & 0 \\
\hline & $L_{6}$ & $1,2,4,7,8,15,12,23,16,31,20,39,24 \ldots$ & $\frac{3 s^{5}+s^{4}+3 s^{3}+2 s^{2}+2 s+1}{(s-1)^{2}(s+1)^{2}}$ & $(-1)^{k}\left(-k+\frac{5}{2}\right)+3 k-\frac{5}{2}$ & 0 \\
\hline & $L_{7}$ & $1,2,4,7,11,15,19,23,27,31,35,39,43 \ldots$ & $\frac{s^{4}+s^{3}+s^{2}+1}{(s-1)^{2}}$ & $4 k-5$ & 0 \\
\hline & $L_{8}$ & $1,2,4,6,8,10,12,14,16,18,20,22,24 \ldots$ & $\frac{s^{2}+1}{(s-1)^{2}}$ & $2 k$ & 0 \\
\hline
\end{tabular}

Continued on next page 
Table 3 - Continued from previous page

\begin{tabular}{|c|c|c|c|c|}
\hline Name & $\begin{array}{l}\text { Degrees } \\
\quad\left\{d_{k}\right\}\end{array}$ & $\begin{array}{l}\text { Generating function } \\
\qquad g(s)\end{array}$ & $\begin{array}{l}\text { Degree fit } \\
\quad d_{k}\end{array}$ & $\begin{array}{l}\text { Entropy } \\
\quad \eta\end{array}$ \\
\hline$L_{9}$ & $1,2,2,5,3,8,4,11,5,14,6,17,7, \ldots$ & $\frac{s^{3}+2 s+1}{(s-1)^{2}(s+1)^{2}}$ & $\frac{(-1)^{k}}{4}(-2 k+1)+k+\frac{3}{4}$ & 0 \\
\hline$L_{10}$ & $1,2,3,5,5,8,7,11,9,14,11,17,13, \ldots$ & $\frac{s^{3}+s^{2}+2 s+1}{(s-1)^{2}(s+1)^{2}}$ & $\frac{(-1)^{k}}{4}(-k+1)+\frac{5 k}{4}+\frac{3}{4}$ & 0 \\
\hline$L_{11}$ & $1,2,4,5,10,8,16,11,22,14,28,17,34, \ldots$ & $\frac{3 s^{4}+s^{3}+2 s^{2}+2 s+1}{(s-1)^{2}(s+1)^{2}}$ & $\frac{(-1)^{k}}{4}(3 k-5)+\frac{9 k}{4}-\frac{3}{4}$ & 0 \\
\hline$L_{12}$ & $1,2,4,5,7,8,10,11,13,14,16,17,19, \ldots$ & $\frac{s^{2}+s+1}{(s-1)^{2}(s+1)}$ & $\frac{(-1)^{k}}{4}+\frac{3 k}{2}+\frac{3}{4}$ & 0 \\
\hline$L_{13}$ & $1,2,4,6,11,10,18,14,25,18,32,22,39, \ldots$ & $\frac{4 s^{4}+2 s^{3}+2 s^{2}+2 s+1}{(s-1)^{2}(s+1)^{2}}$ & $\frac{3(-1)^{k}}{4}(k-2)+\frac{11 k}{4}-\frac{3}{2}$ & 0 \\
\hline$L_{14}$ & $1,2,3,3,6,4,9,5,12,6,15,7,18, \ldots$ & $\frac{s^{4}-s^{3}+s^{2}+2 s+1}{(s-1)^{2}(s+1)^{2}}$ & $\frac{(-1)^{k}}{4}(2 k-3)+k+\frac{3}{4}$ & 0 \\
\hline$L_{15}$ & $1,1,3,3,6,5,9,7,12,9,15,11,18, \ldots$ & $\frac{s^{4}+s^{3}+s^{2}+s+1}{(s-1)^{2}(s+1)^{2}}$ & $\frac{k}{4}\left((-1)^{k}+5\right)$ & 0 \\
\hline$L_{16}$ & $1,2,3,2,5,2,7,2,9,2,11,2,13$, . & $\frac{-2 s^{3}+s^{2}+2 s+1}{(s-1)^{2}(s+1)^{2}}$ & $\frac{(-1)^{k}}{2}(k-1)+\frac{k}{2}+\frac{3}{2}$ & 0 \\
\hline$L_{17}$ & $1,1,3,3,5,5,7,7,9,9,11,11,13, \ldots$ & $\frac{s^{2}+1}{(s-1)^{2}(s+1)}$ & $\frac{(-1)^{k}}{2}+k+\frac{1}{2}$ & 0 \\
\hline$L_{18}$ & $1,2,4,5,11,9,19,13,27,17,35,21,43, \ldots$ & $\frac{s^{6}+s^{5}+4 s^{4}+s^{3}+2 s^{2}+2 s+1}{(s-1)^{2}(s+1)^{2}}$ & $(-1)^{k}(k-2)+3 k-3$ & 0 \\
\hline
\end{tabular}


Table 3 - Continued from previous page

\begin{tabular}{|c|c|c|c|c|}
\hline Name & $\begin{array}{l}\text { Degrees } \\
\quad\left\{d_{k}\right\}\end{array}$ & $\begin{array}{l}\text { Generating function } \\
\qquad g(s)\end{array}$ & $\begin{array}{l}\text { Degree fit } \\
\quad d_{k}\end{array}$ & $\begin{array}{c}\text { Entropy } \\
\eta\end{array}$ \\
\hline$L_{19}$ & $1,2,4,6,11,10,19,14,27,18,35,22,43, \ldots$ & $\frac{s^{6}+4 s^{4}+2 s^{3}+2 s^{2}+2 s+1}{(s-1)^{2}(s+1)^{2}}$ & $(-1)^{k}\left(k-\frac{5}{2}\right)+3 k-\frac{5}{2}$ & 0 \\
\hline$L_{20}$ & $1,1,3,2,6,3,9,4,12,5,15,6,18$. & $\frac{s^{4}+s^{2}+s+1}{(s-1)^{2}(s+1)^{2}}$ & $\frac{(-1)^{k}}{4}(2 k-1)+k+\frac{1}{4}$ & 0 \\
\hline
\end{tabular}




\section{Examples}

In this Section one considers in detail the ${ }_{t} H_{1}^{\varepsilon}$ (4.1a) and ${ }_{1} D_{2}$ (4.2b) equations and shows the explicit form of the quadruple of matrices coming from the CAC, the non-autonomous equations which give the consistency on $\mathbb{Z}^{3}$ and the effective Lax pair. Finally we confirm the predictions of the algebraic entropy analysis showing how they can be explicitly linearized.

\subsection{Example 1: ${ }_{t} H_{1}^{\varepsilon}$}

To construct the Lax Pair for (4.1a) we have to deal with Case 3.10.1 in [10]. The sextuple of equations we consider is:

$$
\begin{aligned}
A & =\alpha_{2}\left(x-x_{1}\right)\left(x_{2}-x_{12}\right)-\alpha_{1}\left(x-x_{2}\right)\left(x_{1}-x_{12}\right) \\
& +\varepsilon^{2} \alpha_{1} \alpha_{2}\left(\alpha_{1}-\alpha_{2}\right), \\
B & =\left(x-x_{2}\right)\left(x_{3}-x_{23}\right)-\alpha_{2}\left(1+\varepsilon^{2} x_{3} x_{23}\right)=0, \\
C & =\left(x-x_{1}\right)\left(x_{3}-x_{13}\right)-\alpha_{1}\left(1+\varepsilon^{2} x_{3} x_{13}\right)=0, \\
\bar{A} & =\alpha_{2}\left(x_{13}-x_{3}\right)\left(x_{123}-x_{23}\right)-\alpha_{1}\left(x_{13}-x_{123}\right)\left(x_{3}-x_{123}\right), \\
\bar{B} & =\left(x_{1}-x_{12}\right)\left(x_{13}-x_{123}\right)-\alpha_{2}\left(1+\varepsilon^{2} x_{13} x_{123}\right)=0, \\
\bar{C} & =\left(x_{2}-x_{12}\right)\left(x_{23}-x_{123}\right)-\alpha_{1}\left(1+\varepsilon^{2} x_{23} x_{123}\right)=0,
\end{aligned}
$$

In this sextuple (4.1a) originates from the $B$ equation.

We now make the following identifications

$$
\begin{array}{lllll}
A: & x \rightarrow u_{p, n} & x_{1} \rightarrow u_{p+1, n} & x_{2} \rightarrow u_{p, n+1} & x_{12} \rightarrow u_{p+1, n+1} \\
B: & x \rightarrow u_{n, m} & x_{2} \rightarrow u_{n+1, m} & x_{3} \rightarrow u_{n, m+1} & x_{23} \rightarrow u_{n+1, m+1} \\
C: & x \rightarrow u_{p, m} & x_{1} \rightarrow u_{p+1, m} & x_{3} \rightarrow u_{p, m+1} & x_{13} \rightarrow u_{p+1, m+1}
\end{array}
$$

so that in any equation we can suppress the dependence on the appropriate parametric variables. On $\mathbb{Z}^{3}$ we get the following triplet of equations:

$$
\begin{aligned}
\tilde{A} & =\alpha_{2}\left(u_{p, n}-u_{p+1, n}\right)\left(u_{p, n+1}-u_{p+1, n+1}\right) \\
& -\alpha_{1}\left(u_{p, n}-u_{p, n+1}\right)\left(u_{p+1, n}-u_{p+1, n+1}\right) \\
& +\varepsilon^{2} \alpha_{1} \alpha_{2}\left(\alpha_{1}-\alpha_{2}\right) F_{m}^{(+)}, \\
\tilde{B} & =\left(u_{n, m}-u_{n+1, m}\right)\left(u_{n, m+1}-u_{n+1, m+1}\right)-\alpha_{2} \\
& -\alpha_{2} \varepsilon^{2}\left(F_{m}^{(+)} u_{n, m+1} u_{n+1, m+1}+F_{m}^{(-)} u_{n, m} u_{n+1, m}\right), \\
\tilde{C} & =\left(u_{p, m}-u_{p+1, m}\right)\left(u_{p, m+1}-u_{p+1, m+1}\right)-\alpha_{1} \\
& -\alpha_{1} \varepsilon^{2}\left(F_{m}^{(+)} u_{p, m+1} u_{p+1, m+1}+F_{m}^{(-)} u_{p, m} u_{p+1, m}\right) .
\end{aligned}
$$

Then with the usual method we find the following Lax Pair:

$$
\begin{aligned}
\tilde{L}_{n, m} & =\left(\begin{array}{cc}
u_{n, m+1} & -u_{n, m} u_{n, m+1}+\alpha_{1} \\
1 & -u_{n, m}
\end{array}\right) \\
& -\varepsilon^{2} \alpha_{1}\left(\begin{array}{cc}
-F_{m}^{(-)} u_{n, m} & 0 \\
0 & F_{m}^{(+)} u_{n, m+1}
\end{array}\right),
\end{aligned}
$$




$$
\begin{aligned}
\tilde{M}_{n, m} & =\left(\begin{array}{cc}
\alpha_{1}\left(u_{n, m}-u_{n+1, m}\right)+\alpha_{2} u_{n+1, m} & -\alpha_{2} u_{n, m} u_{n+1, m} \\
\alpha_{2} & \alpha_{1}\left(u_{n, m}-u_{n+1, m}\right)-\alpha_{2} u_{n, m}
\end{array}\right) \\
& -\varepsilon^{2} \alpha_{1} \alpha_{2}\left(\alpha_{1}-\alpha_{2}\right) F_{m}^{(+)}\left(\begin{array}{cc}
0 & 1 \\
0 & 0
\end{array}\right) .
\end{aligned}
$$

Let us now turn to the linearization procedure. In (4.1a) we must set $\alpha_{2} \neq 0$, otherwise the equation degenerates into $\left(u_{n, m}-u_{n+1, m}\right)\left(u_{n, m+1}-u_{n+1, m+1}\right)=$ 0 , whose solution is trivial on the lattice. Let us define $u_{n, 2 k} \doteq w_{n, k}, u_{n, 2 k+1} \doteq$ $z_{n, k}$; then we have the following system of two coupled autonomous difference equations

$$
\begin{array}{r}
\left(w_{n, k}-w_{n+1, k}\right)\left(z_{n, k}-z_{n+1, k}\right)-\varepsilon^{2} \alpha_{2} z_{n, k} z_{n+1, k}-\alpha_{2}=0 \\
\left(w_{n, k+1}-w_{n+1, k+1}\right)\left(z_{n, k}-z_{n+1, k}\right)-\varepsilon^{2} \alpha_{2} z_{n, k} z_{n+1, k}-\alpha_{2}=0 .
\end{array}
$$

Subtracting (6.5b) to (6.5a), we obtain

$$
\left(w_{n, k}-w_{n+1, k}-w_{n, k+1}+w_{n+1, k+1}\right)\left(z_{n, k}-z_{n+1, k}\right)=0 .
$$

At this point the solution of the system bifurcates:

- Case 1: if $z_{n, k}=f_{k}$, where $f_{k}$ is a generic function of its argument, equation (6.6) is satisfied and from (6.5a) or (6.5b) we have that $\varepsilon \neq 0$ and, solving for $f_{k}$, one gets

$$
f_{k}= \pm \frac{\mathrm{i}}{\varepsilon}
$$

- Case 2: if $z_{n, k} \neq f_{k}$, with $f_{k}$ given in (6.7), one has $w_{n, k}=g_{n}+h_{k}$, where $g_{n}$ and $h_{k}$ are arbitrary functions of their argument. Hence (6.5b) and (6.5a) reduce to

$$
\varepsilon^{2} z_{n, k} z_{n+1, k}+\kappa_{n}\left(z_{n, k}-z_{n+1, k}\right)+1=0, \quad \kappa_{n} \doteq \frac{g_{n+1}-g_{n}}{\alpha_{2}},
$$

so that two sub-cases emerge:

- Sub-case 2.1: if $\varepsilon=0$, (6.8) then $\kappa_{n} \neq 0$, so that, solving,

$$
z_{n+1, k}-z_{n, k}=\frac{1}{\kappa_{n}}
$$

we get

$$
z_{n, k}= \begin{cases}j_{k}+\sum_{l=n_{0}}^{n-1} \frac{1}{\kappa_{l}}, & n \geq n_{0}+1 \\ j_{k}-\sum_{l=n}^{n_{0}-1} \frac{1}{\kappa_{l}}, & n \leq n_{0}-1\end{cases}
$$

where $j_{k}=z_{n_{0}, k}$ is a generic integration function of its argument. 
- Sub-case 2.2: if $\varepsilon \neq 0,(6.8)$ is a discrete Riccati equation which can be linearized by the Möbius transformation $z_{n, k} \doteq \frac{\mathrm{i}}{\varepsilon} \frac{y_{n, k}-1}{y_{n, k}+1}$ to

$$
\left(\mathrm{i} \kappa_{n}-\varepsilon\right) y_{n+1, k}=\left(\mathrm{i} \kappa_{n}+\varepsilon\right) y_{n, k},
$$

which, as $\kappa_{n} \neq \pm \mathrm{i} \varepsilon$ because otherwise $y_{n, k}=0$ and $z_{n, k}=-\mathrm{i} / \varepsilon$. Eq. (6.11) implies

$$
y_{n, k}= \begin{cases}j_{k} \prod_{l=n_{0}}^{n-1} \frac{\mathrm{i} \kappa_{l}+\varepsilon}{\mathrm{i} \kappa_{l}-\varepsilon}, & n \geq n_{0}+1 \\ j_{k} \prod_{l=n}^{n_{0}-1} \frac{\mathrm{i} \kappa_{l}-\varepsilon}{\mathrm{i} \kappa_{l}+\varepsilon}, & n \leq n_{0}-1\end{cases}
$$

where $j_{k}=y_{n_{0}, k}$ is another arbitrary integration function of its argument.

In conclusion we have always completely integrated the original system.

Let us note that in the case $\varepsilon=0$ eq. 4.1a becomes

$$
\left(u_{n, m}-u_{n+1, m}\right)\left(u_{n, m+1}-u_{n+1, m+1}\right)-\alpha_{2}=0,
$$

so that the contact Möbius-type transformation

$$
u_{n+1, m}-u_{n, m}=\sqrt{\alpha_{2}} \frac{1-w_{n, m}}{1+w_{n, m}},
$$

brings (4.1a) into the following first order linear equation:

$$
w_{n, m+1}+w_{n, m}=0,
$$

so that:

$$
u_{n, m}= \begin{cases}k_{m}+\sqrt{\alpha_{2}} \sum_{l=n_{0}}^{l=n-1} \frac{1-(-1)^{m} w_{l}}{1+(-1)^{m} w_{l}}, & n \geq n_{0}+1 \\ k_{m}-\sqrt{\alpha_{2}} \sum_{l=n}^{l=n_{0}-1} \frac{1-(-1)^{m} w_{l}}{1+(-1)^{m} w_{l}}, & n \leq n_{0}-1 .\end{cases}
$$

Here $k_{m}=u_{n_{0}, m}$ and $w_{n}$, are two arbitrary integration functions.

\subsection{Example 2: ${ }_{1} D_{2}$}

Now we consider the equation ${ }_{1} D_{2}(4.2 \mathrm{~b})$. We consider the sextuple of equations given by Case 3.12.2 in [10:

$$
\begin{aligned}
A & =\delta_{2} x+x_{1}+\left(1-\delta_{1}\right) x_{2}+x_{12}\left(x+\delta_{1} x_{2}\right) \\
B & =\left(x-x_{3}\right)\left(x_{2}-x_{23}\right) \\
& +\lambda\left[x+x_{3}-\delta_{1}\left(x_{2}+x_{23}\right)\right]+\delta_{1} \lambda \\
C & =\left(x-x_{3}\right)\left(x_{1}-x_{13}\right)-\lambda\left[\left(\delta_{1} \delta_{2}+\delta_{1}-1\right)\left(x+x_{3}\right)+\delta_{1}\left(x_{1}+x_{13}\right)\right] \\
& +\delta_{1}\left(\delta_{1} \delta_{2}+\delta_{1}-1\right) \lambda^{2} \\
\bar{A} & =\delta_{2} x_{3}+x_{13}+\left(1-\delta_{1}\right) x_{23}+x_{123}\left(x+\delta_{1} x_{23}\right)
\end{aligned}
$$




$$
\begin{aligned}
\bar{B} & =\left(x_{1}-x_{13}\right)\left(x_{12}-x_{123}\right) \\
& +\lambda\left[2 \delta_{2}\left(\delta_{1}-1\right)+\left(\delta_{1}-1-\delta_{1} \delta_{2}\right)-2 \delta_{1} x_{12} x_{123}\right], \\
\bar{C} & =\left(x_{1}-x_{23}\right)\left(x_{12}-x_{123}\right)-\lambda\left(2 \delta_{2}+x_{12}+x_{123}\right) .
\end{aligned}
$$

The triplet of consistent dynamical systems on the $3 D$-lattice is:

$$
\begin{aligned}
& \tilde{A} \doteq \\
& \doteq\left(F_{p+n}^{(-)}-\delta_{1} F_{p}^{(+)} F_{n}^{(-)}+\delta_{2} F_{p}^{(+)} F_{n}^{(+)}\right) u_{p, n}+\left(F_{p+n}^{(+)}-\delta_{1} F_{p}^{(-)} F_{n}^{(-)}+\delta_{2} F_{p}^{(-)} F_{n}^{(+)}\right) u_{p+1, n}+ \\
& +\left(F_{p+n}^{(+)}-\delta_{1} F_{p}^{(+)} F_{n}^{(+)}+\delta_{2} F_{p}^{(+)} F_{n}^{(-)}\right) u_{p, n+1}+\left(F_{p+n}^{(-)}-\delta_{1} F_{p}^{(-)} F_{n}^{(+)}+\delta_{2} F_{p}^{(-)} F_{n}^{(-)}\right) u_{p+1, n+1}+ \\
& +\delta_{1}\left(F_{n}^{(-)} u_{p, n} u_{p+1, n}+F_{n}^{(+)} u_{p, n+1} u_{p+1, n+1}\right)+F_{p+n}^{(+)} u_{p, n} u_{p+1, n+1}+F_{p+n}^{(-)} u_{p+1, n} u_{p, n+1}, \\
& \tilde{B} \doteq \\
& \doteq \lambda\left\{\left[\left(\delta_{1}-1\right) F_{n}^{(+)}-\delta_{1}\right] F_{p}^{(+)}+\left(\delta_{1}-1-\delta_{1} \delta_{2}\right) F_{p}^{(-)} F_{n}^{(-)}\right\}\left(u_{n, m}+u_{n, m+1}\right)+ \\
& +\lambda\left\{\left[\left(\delta_{1}-1\right) F_{n}^{(-)}-\delta_{1}\right] F_{p}^{(+)}+\left(\delta_{1}-1-\delta_{1} \delta_{2}\right) F_{p}^{(-)} F_{n}^{(+)}\right\}\left(u_{n+1, m}+u_{n+1, m+1}\right)- \\
& -2 \delta_{1} \lambda F_{p}^{(-)}\left(F_{n}^{(-)} u_{n, m} u_{n, m+1}+F_{n}^{(+)} u_{n+1, m} u_{n+1, m+1}\right)+ \\
& +\left(u_{n, m}-u_{n, m+1}\right)\left(u_{n+1, m}-u_{n+1, m+1}\right)+\delta_{1} \lambda^{2} F_{p}^{(+)}+2\left(\delta_{1}-1\right) \delta_{2} \lambda F_{p}^{(-)}, \\
& \tilde{C} \doteq \\
& \doteq \lambda\left\{\left[\left(1-\delta_{1} \delta_{2}\right) F_{p}^{(+)}-\delta_{1}\right] F_{n}^{(+)}+F_{p}^{(-)} F_{n}^{(-)}\right\}\left(u_{p, m}+u_{p, m+1}\right)+2 \delta_{2} \lambda F_{n}^{(-)} \\
& +\lambda\left\{\left[\left(1-\delta_{1} \delta_{2}\right) F_{p}^{(-)}-\delta_{1}\right] F_{n}^{(+)}+F_{p}^{(+)} F_{n}^{(-)}\right\}\left(u_{p+1, m}+u_{p+1, m+1}\right)+ \\
& +\left(u_{p, m}-u_{p, m+1}\right)\left(u_{p+1, m}-u_{p+1, m+1}\right)+\delta_{1}\left(\delta_{1}-1+\delta_{1} \delta_{2}\right) \lambda^{2} F_{n}^{(+)} .
\end{aligned}
$$

We leave out the Lax pair for ${ }_{1} D_{2}$ as they are too complicate to write down and not worth while the effort for the reader. If necessary one can always write them down using the standard procedure outlined above.

Let us now turn to the linearization procedure. Notice that there is no combination of the parameters $\delta_{1}$ and $\delta_{2}$ such that (4.2b) becomes non-autonomous. So we are naturally induced to introduce the following four fields:

$$
\begin{gathered}
w_{s, t}=u_{2 s, 2 t}, \quad y_{s, t}=u_{2 s+1,2 t}, \\
v_{s, t}=u_{2 s, 2 t+1} \quad z_{s, t}=u_{2 s+1,2 t+1} .
\end{gathered}
$$

which transform (4.2b) into the following system of four coupled autonomous difference equations:

$$
\begin{aligned}
\left(1-\delta_{1}\right) v_{s, t}+\delta_{2} w_{s, t}+y_{s, t}+\left(\delta_{1} v_{s, t}+w_{s, t}\right) z_{s, t} & =0 \\
\left(1-\delta_{1}\right) v_{s+1, t}+\delta_{2} w_{s+1, t}+y_{s, t}+\left(\delta_{1} v_{s+1, t}+w_{s+1, t}\right) z_{s, t} & =0 \\
\left(1-\delta_{1}\right) v_{s, t}+\delta_{2} w_{s, t+1}+y_{s, t+1}+\left(\delta_{1} v_{s, t}+w_{s, t+1}\right) z_{s, t} & =0 \\
\left(1-\delta_{1}\right) v_{s+1, t}+\delta_{2} w_{s+1, t+1}+y_{s, t+1}+\left(\delta_{1} v_{s+1, t}+w_{s+1, t+1}\right) z_{s, t} & =0 .
\end{aligned}
$$

Let us solve (6.20a) with respect to $y_{s, t}$ :

$$
y_{s, t}=-\left(1-\delta_{1}\right) v_{s, t}-\delta_{2} w_{s, t}-\left(\delta_{1} v_{s, t}+w_{s, t}\right) z_{s, t}
$$

and let us insert $y_{s, t}$ into (6.20b) in order to get an equation solvable for $z_{s, t}$. This is possible iff $\delta_{1} v_{s, t}+w_{s, t} \neq f_{t}$, with $f_{t}$ a generic function of $t$, since in 
this case the coefficient of $z_{s, t}$ is zero. Then the solution of the system (6.20) bifurcates.

Case 1 Assume that $\delta_{1} v_{s, t}+w_{s, t} \neq f_{t}$, then we can solve with respect to $z_{s, t}$ the expression obtained inserting (6.21) into (6.20b). We get:

$$
z_{s, t}=-\frac{\left(1-\delta_{1}\right)\left(v_{s+1, t}-v_{s, t}\right)+\delta_{2}\left(w_{s+1, t}-w_{s, t}\right)}{\delta_{1}\left(v_{s+1, t}-v_{s, t}\right)+w_{s+1, t}-w_{s, t}} .
$$

Now we can substitute (6.21) and (6.22) together with their difference consequences into (6.20c) and (6.20d) and we get two equations for $w_{s, t}$ and $v_{s, t}$ :

$$
\begin{aligned}
\left(\delta_{1} \delta_{2}+\delta_{1}-1\right)[ & w_{s+1, t+1} v_{s, t+1} w_{s, t}+v_{s+1, t+1} w_{s, t+1} w_{s+1, t} \\
& -v_{s+1, t+1} w_{s, t+1} w_{s, t}+w_{s, t+1} v_{s, t} w_{s+1, t+1} \\
& +v_{s, t} w_{s+1, t+1} w_{s+1, t}-w_{s, t+1} v_{s+1, t} w_{s+1, t+1} \\
& -w_{s, t+1}^{2} v_{s, t}+w_{s, t+1}^{2} v_{s+1, t} \\
& -w_{s+1, t+1} v_{s, t+1} w_{s+1, t}-v_{s, t} w_{s+1, t+1} w_{s, t} \\
& -v_{s, t} w_{s, t+1} w_{s+1, t}+v_{s, t} w_{s, t+1} w_{s, t} \\
& -\delta_{1}\left(v_{s, t} v_{s, t+1} w_{s+1, t}+w_{s, t+1} v_{s, t} v_{s, t+1}\right. \\
& -w_{s+1, t+1} v_{s, t+1} v_{s, t}+w_{s+1, t+1} v_{s, t+1} v_{s+1, t} \\
& +v_{s, t} v_{s+1, t+1} w_{s, t}-v_{s, t} v_{s+1, t+1} w_{s+1, t} \\
& \left.\left.-v_{s, t} v_{s, t+1} w_{s, t}-w_{s, t+1} v_{s+1, t} v_{s, t+1}\right)\right] \\
\left(\delta_{1} \delta_{2}+\delta_{1}-1\right)[ & w_{s+1, t+1}^{2} v_{s+1, t}+w_{s+1, t+1} v_{s, t+1} w_{s+1, t} \\
& -w_{s+1, t+1} v_{s, t+1} w_{s, t}-w_{s+1, t+1}^{2} v_{s, t} \\
& -w_{s, t+1} v_{s+1, t} w_{s+1, t+1}+w_{s, t+1} v_{s, t} w_{s+1, t+1} \\
& -v_{s+1, t+1} w_{s, t+1} w_{s+1, t}+v_{s+1, t} w_{s, t+1} w_{s+1, t} \\
& +v_{s+1, t+1} w_{s, t+1} w_{s, t}-v_{s+1, t} w_{s+1, t+1} w_{s+1, t} \\
& +v_{s+1, t} w_{s+1, t+1} w_{s, t}-v_{s+1, t} w_{s, t+1} w_{s, t}+ \\
& \delta_{1}\left(-v_{s+1, t} v_{s+1, t+1} w_{s+1, t}+v_{s+1, t} v_{s+1, t+1} w_{s, t}\right. \\
& -w_{s+1, t+1} v_{s, t} v_{s+1, t+1}-v_{s+1, t} v_{s, t+1} w_{s, t} \\
& -v_{s+1, t+1} w_{s, t+1} v_{s+1, t}+w_{s+1, t+1} v_{s+1, t} v_{s+1, t+1} \\
& \left.+v_{s+1, t} v_{s, t+1} w_{s+1, t}+v_{s+1, t+1} w_{s, t+1} v_{s, t}\right]
\end{aligned}
$$

If

$$
\delta_{1}\left(1+\delta_{2}\right)=1,
$$

then (6.23) are identically satisfied. If $\delta_{1}\left(1+\delta_{2}\right) \neq 1$, adding (6.23a) and $6.23 \mathrm{~b}$, we obtain:

$$
\begin{aligned}
& \left(w_{s, t}-w_{s+1, t}-w_{s, t+1}+w_{s+1, t+1}\right) . \\
& \left(v_{s+1, t}-v_{s, t}\right)\left(\delta_{1}+\delta_{1} \delta_{2}-1\right)=0 .
\end{aligned}
$$

Supposing $\delta_{1}+\delta_{1} \delta_{2} \neq 1$ we can annihilate the first or the second factor. If set equal to zero the second factor, we get $v_{s, t}=g_{t}$ with $g_{t}$ arbitrary function of $t$ alone. Substituting this result into(6.23a or (6.23b), they 
are identically satisfied provided $g_{t}=g_{0}$, with $g_{0}$ constant. Then the only non-trivial case is when $\delta_{1}+\delta_{1} \delta_{2} \neq 1$, and $v_{s, t} \neq g_{t}$. In this case we have that $w_{s, t}$ solves the discrete wave equation, i.e. $w_{s, t}=h_{s}+l_{t}$. Substituting $w_{s, t}$ into (6.23) we get a single equation for $v_{s, t}$ :

$$
\begin{aligned}
\left(h_{s}-h_{s+1}\right)[ & \left(v_{s+1, t+1}-v_{s+1, t}\right)\left(h_{s}+l_{t+1}\right) \\
& -\left(v_{s, t+1}-v_{s, t}\right)\left(h_{s+1}+l_{t+1}\right) \\
& \left.+\delta_{1}\left(v_{s, t} v_{s+1, t+1}-v_{s+1, t} v_{s, t+1}\right)\right]=0 .
\end{aligned}
$$

which is identically satisfied if $h_{s}=h_{0}$, with $h_{0}$ a constant. Therefore we have a non-trivial cases only if $h_{s} \neq h_{0}$.

Case 1.1 We have a great simplification if in addition to $h_{s} \neq h_{0}$ we have $\delta_{1}=0$. In this case (6.26) is linear:

$$
\begin{aligned}
\left(v_{s+1, t+1}-v_{s+1, t}\right) & \left(h_{s}+l_{t+1}\right) \\
& -\left(v_{s, t+1}-v_{s, t}\right)\left(h_{s+1}+l_{t+1}\right)=0 .
\end{aligned}
$$

Eq. (6.27) can be easily integrated twice to give:

$$
v_{s, t}= \begin{cases}j_{s}+\sum_{k=t_{0}}^{t-1}\left(h_{s}+l_{k+1}\right) i_{k}, & t \geq t_{0}+1, \\ j_{s}-\sum_{k=t}^{t_{0}-1}\left(h_{s}+l_{k+1}\right) i_{k}, & t \leq t_{0}-1,\end{cases}
$$

with $i_{t}$ and $j_{s}=v_{s, t_{0}}$ arbitrary integration functions.

Case 1.2 Now let us suppose again $h_{s} \neq h_{0}, \delta_{1} \neq 0$ but let us choose $l_{t}=l_{0}$, with $l_{0}$ a constant. Performing the translation $\theta_{s, t}=v_{s, t}+$ $\left(h_{s}+l\right) / \delta_{1}$, from (6.26) we get:

$$
\theta_{s, t} \theta_{s+1, t+1}-\theta_{s+1, t} \theta_{s, t+1}=0 .
$$

Eq. (6.29) is linearizable via a Cole-Hopf transformation $\Theta_{s, t}=$ $\theta_{s+1, t} / \theta_{s, t}$ as $v_{s, t}$ cannot be identically zero. This linearization yields the general solution $\theta_{s, t}=S_{s} T_{t}$ with $S_{s}$ and $T_{t}$ arbitrary functions of their argument.

Case 1.3 Finally if $h_{s} \neq h, \delta_{1} \neq 0$ and $l_{t} \neq l_{0}$, we perform the transformation

$$
\theta_{s, t}=\frac{1}{\delta_{1}}\left[\left(l_{t}-l_{t+1}\right) v_{s, t}-h_{s}-l_{t+1}\right] .
$$

Then from (6.26) we get:

$$
\theta_{s, t}\left(1+\theta_{s+1, t+1}\right)-\theta_{s+1, t}\left(1+\theta_{s, t+1}\right)=0,
$$

which, as $v_{s, t}$ cannot be identically zero, is easily linearized via the Cole-Hopf transformation $\Theta_{s, t}=\left(1+\theta_{s, t+1}\right) / \theta_{s, t}$ to $\Theta_{s+1, s}-\Theta_{s, t}=0$ which yields for $\theta_{s, t}$ the linear equation:

$$
\theta_{s, t+1}-p_{t} \theta_{s, t}+1=0,
$$

where $p_{t}$ is an arbitrary integration function. Then the general solution is given by:

$$
\theta_{s, t}=\left\{\begin{array}{c}
\left(u_{s}-\sum_{l=t_{0}}^{t-1} \prod_{j=t_{0}}^{l} p_{j}^{-1}\right) \prod_{k=t_{0}}^{t-1} p_{k}, \quad t \geq t_{0}+1, \\
u_{s} \prod_{k=t}^{t_{0}-1} p_{k}^{-1}+\sum_{l=t}^{t_{0}-1} \prod_{j=t}^{l} p_{j}^{-1}, \quad t \leq t_{0}-1,
\end{array}\right.
$$

where $u_{s}=\theta_{s, t_{0}}$ is an arbitrary integration function. 
Case 2 We now suppose:

$$
w_{s, t}=f_{t}-\delta_{1} v_{s, t},
$$

where $f_{t}$ is a generic function of its argument. Inserting (6.21) and (6.34) and their difference consequences into (6.20), we get:

$$
\left(v_{s+1, t}-v_{s, t}\right)\left(\delta_{1}+\delta_{1} \delta_{2}-1\right)=0,
$$

and the two relations:

$$
\begin{aligned}
f_{t+1} z_{s, t+1} & +\left[\delta_{1}\left(v_{s, t}-v_{s, t+1}\right)-f_{t+1}\right] z_{s, t} \\
+ & \left(\delta_{1}-1\right)\left(v_{s, t}-v_{s, t+1}\right) \\
f_{t+1} z_{s, t+1} & +\left[\delta_{1}\left(v_{s+1, t}-v_{s+1, t+1}\right)-f_{t+1}\right] z_{s, t} \\
+ & \left(\delta_{1}-1\right)\left(v_{s+1, t}-v_{s, t+1}\right)+\delta_{1} \delta_{2}\left(v_{s+1, t+1}-v_{s, t+1}\right) .
\end{aligned}
$$

Hence in (6.35) we have a biforcation.

Case 2.1 If we annihilate the second factor in (6.35) we get $\delta_{1}\left(1+\delta_{2}\right)=$ 1, i.e. $\delta_{1} \neq 0$. Then adding (6.36a) and (6.36b) we obtain:

$$
\left(v_{s, t}-v_{s+1, t}-v_{s, t+1}+v_{s+1, t+1}\right)\left(1-\delta_{1}+\delta_{1} z_{s, t}\right)=0 .
$$

It seems that we are facing a new bifurcation. However annihilating the second factor, i.e. assuming that $z_{s, t}=1-1 / \delta_{1}$ give a trivial case, since (6.36) are identically satisfied. Therefore we may assume that $z_{s, t} \neq 1-1 / \delta_{1}$. This implies that $v_{s, t}=h_{s}+k_{t}$, where $h_{s}$ and $k_{t}$ are generic integration functions of their argument. Inserting it in (6.36) we can obtain the following linear equation for $z_{s, t}$ :

$$
f_{t+1} z_{s, t+1}+\left(\delta_{1} j_{t}-f_{t+1}\right) z_{s, t}+\left(\delta_{1}-1\right) j_{t}=0,
$$

with $j_{t}=k_{t+1}-k_{t}$. This equation can be solved it gives:

$$
\begin{aligned}
& z_{s, t}=(-1)^{t}\left(\delta_{1}-1\right) \prod_{t^{\prime}=0}^{t-1} \frac{\delta_{1} j_{t^{\prime}}-f_{t^{\prime}+1}}{f_{t^{\prime}+1}} . \\
& \sum_{t^{\prime \prime}=0}^{t-1} \frac{j_{t^{\prime \prime}}(-1)^{t^{\prime \prime}}}{f_{t^{\prime \prime}+1} \prod_{t^{\prime}=0}^{t^{\prime \prime}} \frac{\delta_{1} j_{t^{\prime}}-f_{t^{\prime}+1}}{f_{t^{\prime}+1}}} \\
&+(-1)^{t} z_{s, 0} \prod_{t^{\prime}=0}^{t-1} \frac{\delta_{1} j_{t^{\prime}}-f_{t^{\prime}+1}}{f_{t^{\prime}+1}}
\end{aligned}
$$

Case 2.2 Now we annihilate the first factor in (6.35) i.e. $\delta_{1}\left(1+\delta_{2}\right) \neq 1$ and $v_{s, t}=l_{t}$, where $l_{t}$ is an arbitrary function of its argument. From (6.36) we obtain (6.38) with $j_{t}=l_{t+1}-l_{t}$.

In conclusion we have always integrated the original system using an explicit linearization through a series of transformations and biforcations.

As a final remark we observe that every transformation used in the linearization procedure both for the ${ }_{t} H_{1}^{\varepsilon}$ (4.1a) equation and for the ${ }_{1} D_{2}$ (4.2b equation is 
bi-rational in the fields and their shifts (like the Cole-Hopf-type transformations). This, in fact, has to be expected, since the Algebraic Entropy test is valid only if we allow transformations which preserve the algebrogeometric structure underlying the evolution procedure [30. Indeed there are examples on one-dimensional lattice of equations chaotic according to the Algebraic Entropy, but linearizable using some transcendental transformations [15]. So exhibiting the explicit linearization and showing that it can be attained by bi-rational transformations is indeed a very strong evidence of the Algebraic Entropy conjecture 22]. Indeed this does not prevent the fact that in some cases such equations can be linearized through some transcendental transformations. In fact if $\varepsilon=0$ the ${ }_{1} H_{1}^{\varepsilon}$ equation (4.1a) can be linearized through the transcendental contact transformation:

$$
u_{n, m}-u_{n+1, m}=\sqrt{\alpha_{2}} e^{z_{n, m}},
$$

i.e.

$$
z_{n, m}=\log \frac{u_{n, m}-u_{n+1, m}}{\sqrt{\alpha_{2}}},
$$

with log standing for the principal value of the complex logarithm (the principal value is intended for the square root too). The transformation (6.40) brings (4.1a) into the following family of first order linear equations:

$$
z_{n, m+1}+z_{n, m}=2 \mathrm{i} \pi \kappa, \quad \kappa=0,1 .
$$

However this kind of transformation does not prove the result of the Algebraic Entropy and the method explained in Section 6.1 should be considered the correct one.

\section{Conclusions}

In this paper we have firstly identified all independent nonlinear, consistent quad-equations on a single cell not of type $Q$ or rhombic $H^{4}$ up to (Möb) ${ }^{4}$ transformations on the fields and rotations, translations and inversions of the reference frame.

Then we have established an automorphism between the group of (Möb $)^{4}$ and what we have called the nonautonomous lifting of (Möb) ${ }^{4}$. This result enabled us to construct for each of the quad-equation on a single cell the corresponding dynamical system on a 2D-lattice according to the Boll extension procedure. Then we present all independent, nonlinear 2D-dynamical systems which are consistent on the 3D-lattice up to the stated lifting, rotations, translations and inversions of the discrete indexes.

For any dynamical system we performed an algebraic entropy analysis which suggested the possibility to linearize all the listed non autonomous equations. Two examples were worked in detail to confirm the algebraic entropy findings.

We are working now to show the effective linearizability and Darboux integrability of all the systems we have listed 18. Among the open questions is the use that can be made of the Lax pairs: can it be of some help to show linearization? Moreover, is it possible to demonstrate they are indeed fake Lax pairs [12,13]? Some results in this direction for the ${ }_{t} H_{1}^{\varepsilon}$ equation are presented in [19]. 


\section{Acknowledgments}

CS and DL have been partly supported by the Italian Ministry of Education and Research, 2010 PRIN Continuous and discrete nonlinear integrable evolutions: from water waves to symplectic maps.

GG and DL are supported by INFN IS-CSN4 Mathematical Methods of Nonlinear Physics.

\section{Appendix}

\section{A Construction of the lattice equation and of its Lax pair}

Let a consider a quad equation $Q=Q\left(x, x_{1}, x_{2}, x_{12} ; \alpha_{1}, \alpha_{2}\right)$ of the ones introduced in Theorem 1. An equation of this kind, even if consistent around the cube, if not treated with care can turn into a non integrable equation. For example, let us consider the deformed $H_{1}$ equation, ${ }_{r} H_{1}^{\varepsilon}[3$ :

$$
\left(x-x_{12}\right)\left(x_{1}-x_{2}\right)-\left(\alpha_{1}-\alpha_{2}\right)\left(1+\varepsilon^{2} x_{1} x_{2}\right)=0,
$$

and let us consider the trivial embedding of such equation into a lattice given by the identification?

$$
x \rightarrow u_{n, m}, \quad x_{1} \rightarrow u_{n+1, m}, \quad x_{2} \rightarrow u_{n, m+1}, \quad x_{12} \rightarrow u_{n+1, m+1} .
$$

Eq. (A.1) with the identification (A.2) becomes the following lattice equation:

$$
\left(u_{n, m}-u_{n+1, m+1}\right)\left(u_{n+1, m}-u_{n, m+1}\right)-\left(\alpha_{1}-\alpha_{2}\right)\left(1+\varepsilon^{2} u_{n+1, m} u_{n, m+1}\right)=0 .
$$

We apply the algebraic entropy test 4 to $(\mathrm{A} .3$ ) and we find the following growth in the Nord-East $(-,+)$ direction of the degrees:

$$
\left\{d_{-,+}\right\}=\{1,2,4,9,21,50,120,289 \ldots\} .
$$

This sequence has generating function

$$
g_{-,+}=\frac{1-s-s^{2}}{s^{3}+s^{2}-3 s+1}
$$

and therefore has a non-zero algebraic entropy given by:

$$
\eta_{-,+}=\log (1+\sqrt{2})
$$

corresponding to the entropy of a non-integrable lattice equation.

The identification (A.2) is not the only possible embedding. An embedding of (A.1) into a $\mathbb{Z}^{2}$ lattice is obtained by choosing an elementary cell of dimension

\footnotetext{
${ }^{3}$ Generally from now we will call the field variables $u$ to distinguish them from the "static" vertex indices $x$

${ }^{4}$ For more details on degree of growth, algebraic entropy and related subjects see Section 5 and references therein.
} 
greater than one as the one depicted in Fig. 3. In such a case A.1 can be extended to a lattice and we will get a different partial difference equation. Since a priori $Q \neq|Q \neq Q \neq| Q$ the obtained lattice will be a four color lattice, see Fig. 3. Choosing the origin on the $\mathbb{Z}^{2}$ lattice in the point $x$ we obtain a lattice equation of the following form:

$$
\widetilde{Q}[u]= \begin{cases}Q\left(u_{n, m}, u_{n+1, m}, u_{n, m+1}, u_{n+1, m+1}\right) & n=2 k, m=2 k, k \in \mathbb{Z}, \\ \mid Q\left(u_{n, m}, u_{n+1, m}, u_{n, m+1}, u_{n+1, m+1}\right) & n=2 k+1, m=2 k, k \in \mathbb{Z}, \\ \underline{Q}\left(u_{n, m}, u_{n+1, m}, u_{n, m+1}, u_{n+1, m+1}\right) & n=2 k, m=2 k+1, k \in \mathbb{Z}, \\ \underline{\mid Q}\left(u_{n, m}, u_{n+1, m}, u_{n, m+1}, u_{n+1, m+1}\right) & n=2 k+1, m=2 k+1, k \in \mathbb{Z},\end{cases}
$$

We could have constructed $\widetilde{Q}[u]$ starting from any other point in Fig. 3 as the origin, but such equations would differ from each other only by a translation, a rotation or a reflection. So in the sense of Theorem 1 they will be equivalent to (A.7).

In the case of ${ }_{r} H_{1}^{\varepsilon}$ we have:

$$
{ }_{r} \widetilde{H}_{1}^{\varepsilon}=\left\{\begin{array}{cl}
\left(u_{n, m}-u_{n+1, m+1}\right)\left(u_{n+1, m}-u_{n, m+1}\right) & |n|+|m|=2 k, \quad k \in \mathbb{Z}, \\
-\left(\alpha_{1}-\alpha_{2}\right)\left(1+\varepsilon^{2} u_{n+1, m} u_{n, m+1}\right), & \\
\left(u_{n, m}-u_{n+1, m+1}\right)\left(u_{n+1, m}-u_{n, m+1}\right) & |n|+|m|=2 k+1, \quad k \in \mathbb{Z}, \\
-\left(\alpha_{1}-\alpha_{2}\right)\left(1+\varepsilon^{2} u_{n, m} u_{n+1, m+1}\right), &
\end{array}\right.
$$

where we have used the symmetry properties of the equation ${ }_{r} H_{1}^{\varepsilon}$. This result coincide with that presented in 31.

We shall now consider quad equations which satisfy CAC, since we are concerned about integrability. So let us consider six-tuples of quad equations (1.4) assigned to the faces of a 3D cube as displayed in Fig. 2.

First let us notice that, without loss of generality, we can assume that, if $Q$ is the consistent quad equation we are interested in, then we may assume that $Q$ is the bottom equation i.e. $Q=A$. Indeed if we are interested in an equation on the side of the cube of Fig. 2 and these equations are different from $A$ (once made the appropriate substitutions) we may just rotate it and re-label the vertices in an appropriate manner, so that our side equation will become the bottom equation. In this way following again 8 and taking into account the result stated above we may build an embedding in $\mathbb{Z}^{3}$, whose points we shall label as triples $(n, m, p)$, of the consistency cube. To this end we reflect the consistency cube with respect to the normal of the back and the right side and then complete again with another reflection, just in the same way we did for the square. Using the same notations as in the planar case we see which are the proper equations which must be put on the sides of the "multicube". Their form can therefore be described as in (A.7). As a result we end up with Fig. 6. where the functions appearing on the top and on the bottom can be defined as in A.7 5 while on the sides we shall have:

$$
\begin{aligned}
& \underline{B}\left(x, x_{2}, x_{3}, x_{23}\right)=B\left(x_{2}, x, x_{23}, x_{3}\right), \\
& \underline{\bar{B}}\left(x_{1}, x_{12}, x_{13}, x_{123}\right)=\bar{B}\left(x_{12}, x_{1}, x_{123}, x_{13}\right), \\
& \mid C\left(x, x_{1}, x_{3}, x_{13}\right)=C\left(x_{1}, x, x_{13}, x_{3}\right),
\end{aligned}
$$

\footnotetext{
${ }^{5}$ Obviously in the case of $\bar{A}$ one should traslate every point by one in the $p$ direction.
} 


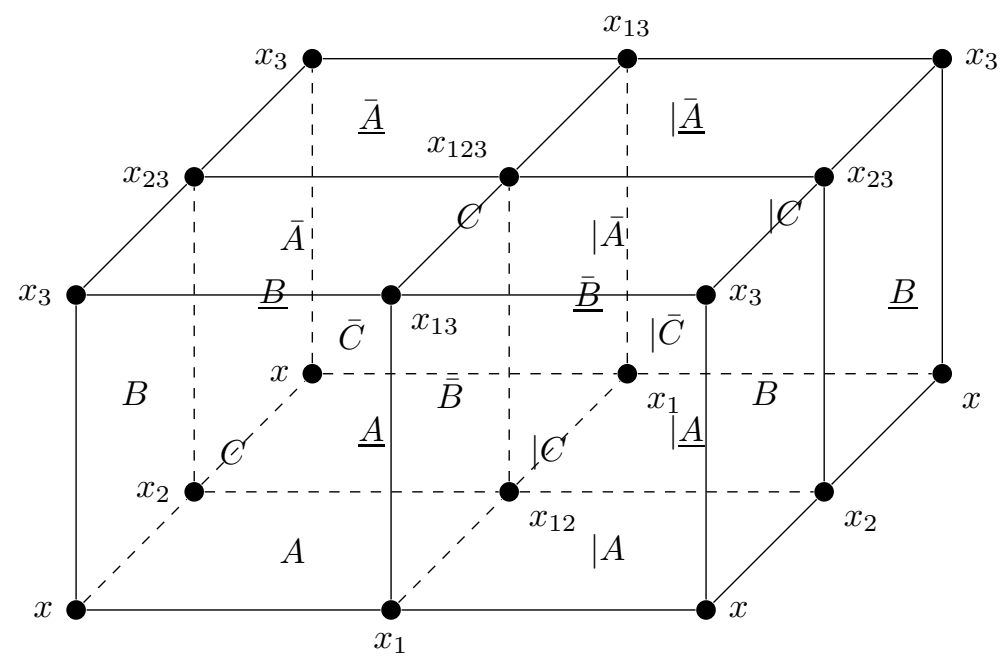

Figure 6: The extension of the consistency cube.

$$
\mid \bar{C}\left(x_{2}, x_{12}, x_{23}, x_{123}\right)=\bar{C}\left(x_{12}, x_{2}, x_{123}, x_{23}\right) .
$$

From (A.9) we obtain the analogous in $\mathbb{Z}^{3}$ of A.7). We have a new consistency cube, see Fig. 6, with equations given by 6 :

$$
\begin{aligned}
& \tilde{\bar{A}}[u]=\left\{\begin{array}{l}
\bar{A}\left(u_{n, m, p+1}, u_{n+1, m, p+1}, u_{n, m+1, p+1}, u_{n+1, m+1, p+1}\right), \\
\mid \bar{A}\left(u_{n, m, p+1}, u_{n+1, m, p+1}, u_{n, m+1, p+1}, u_{n+1, m+1, p+1}\right), \\
\overline{\bar{A}}\left(u_{n, m, p+1}, u_{n+1, m, p+1}, u_{n, m+1, p+1}, u_{n+1, m+1, p+1}\right), \\
\mid \bar{A}\left(u_{n, m, p+1}, u_{n+1, m, p+1}, u_{n, m+1, p+1}, u_{n+1, m+1, p+1}\right),
\end{array}\right. \\
& \widetilde{B}[u]=\left\{\begin{array}{l}
B\left(u_{n, m, p}, u_{n, m+1, p}, u_{n, m, p+1}, u_{n, m+1, p+1}\right), \\
\bar{B}\left(u_{n, m, p}, u_{n, m+1, p}, u_{n, m, p+1}, u_{n, m+1, p+1}\right), \\
\underline{B}\left(u_{n, m, p}, u_{n, m+1, p}, u_{n, m, p+1}, u_{n, m+1, p+1}\right), \\
\bar{B}\left(u_{n, m, p}, u_{n, m+1, p}, u_{n, m, p+1}, u_{n, m+1, p+1}\right),
\end{array}\right. \\
& \widetilde{\bar{B}}[u]=\left\{\begin{array}{l}
\bar{B}\left(u_{n+1, m, p}, u_{n+1, m+1, p}, u_{n+1, m, p+1}, u_{n+1, m+1, p+1}\right), \\
B\left(u_{n+1, m, p}, u_{n+1, m+1, p}, u_{n+1, m, p+1}, u_{n+1, m+1, p+1}\right), \\
\bar{B}\left(u_{n+1, m, p}, u_{n+1, m+1, p}, u_{n+1, m, p+1}, u_{n+1, m+1, p+1}\right), \\
\underline{B}\left(u_{n+1, m, p}, u_{n+1, m+1, p}, u_{n+1, m, p+1}, u_{n+1, m+1, p+1}\right),
\end{array}\right. \\
& \widetilde{C}[u]=\left\{\begin{array}{l}
C\left(u_{n, m, p}, u_{n+1, m, p}, u_{n, m, p+1}, u_{n+1, m, p+1}\right), \\
\mid C\left(u_{n, m, p}, u_{n+1, m, p}, u_{n, m, p+1}, u_{n+1, m, p+1}\right), \\
\bar{C}\left(u_{n, m, p}, u_{n+1, m, p}, u_{n, m, p+1}, u_{n+1, m, p+1}\right), \\
\bar{C}\left(u_{n, m, p}, u_{n+1, m, p}, u_{n, m, p+1}, u_{n+1, m, p+1}\right),
\end{array}\right.
\end{aligned}
$$

${ }^{6}$ For the sake of the semplicity of the presentation we have left out if $n$ and $m$ are even or odd integers. They are recovered by comparing with A.7. 


$$
\widetilde{\bar{C}}[u]=\left\{\begin{array}{l}
\bar{C}\left(u_{n, m+1, p}, u_{n+1, m+1, p}, u_{n, m+1, p+1}, u_{n+1, m+1, p+1}\right), \\
\mid \bar{C}\left(u_{n, m+1, p}, u_{n+1, m+1, p}, u_{n, m+1, p+1}, u_{n+1, m+1, p+1}\right), \\
C\left(u_{n, m, p}, u_{n+1, m+1, p}, u_{n, m+1, p+1}, u_{n+1, m+1, p+1}\right), \\
\mid \bar{C}\left(u_{n, m+1, p}, u_{n+1, m+1, p}, u_{n, m+1, p+1}, u_{n+1, m,+1 p+1}\right),
\end{array}\right.
$$

This means that the "multicube" of Fig. 6 appears as the usual consistency cube of Fig. 2 with the following identifications:

$$
A \rightsquigarrow \widetilde{A}, \quad \bar{A} \rightsquigarrow \widetilde{\bar{A}}, \quad B \rightsquigarrow \widetilde{B}, \quad \bar{B} \rightsquigarrow \widetilde{\bar{B}}, \quad C \rightsquigarrow \widetilde{C}, \quad \bar{C} \rightsquigarrow \widetilde{\bar{C}} .
$$

As an example of this procedure, let us consider again the equation ${ }_{r} H_{1}^{\varepsilon} \cdot{ }_{r} H_{1}^{\varepsilon}$ has the CAC equations:

$$
\begin{aligned}
A & =\left(x-x_{12}\right)\left(x_{1}-x_{2}\right)+\left(\alpha_{1}-\alpha_{2}\right)\left(1+\varepsilon x_{1} x_{2}\right), \\
\bar{A} & =\left(x_{3}-x_{123}\right)\left(x_{13}-x_{23}\right)+\left(\alpha_{1}-\alpha_{2}\right)\left(1+\varepsilon x_{3} x_{123}\right), \\
B & =\left(x-x_{23}\right)\left(x_{2}-x_{3}\right)+\left(\alpha_{2}-\alpha_{3}\right)\left(1+\varepsilon x_{2} x_{3}\right), \\
\bar{B} & =\left(x_{1}-x_{123}\right)\left(x_{12}-x_{13}\right)+\left(\alpha_{2}-\alpha_{3}\right)\left(1+\varepsilon x_{1} x_{123}\right), \\
C & =\left(x-x_{13}\right)\left(x_{1}-x_{3}\right)+\left(\alpha_{1}-\alpha_{3}\right)\left(1+\varepsilon x_{1} x_{3}\right), \\
\bar{C} & =\left(x_{2}-x_{123}\right)\left(x_{12}-x_{23}\right)+\left(\alpha_{1}-\alpha_{2}\right)\left(1+\varepsilon x_{2} x_{123}\right),
\end{aligned}
$$

therefore from A.10, A.11 we get the following consistency on the "multicube":

$$
\begin{aligned}
& A=\left\{\begin{array}{cl}
\left(u_{n, m, p}-u_{n+1, m+1, p}\right)\left(u_{n+1, m, p}-u_{n, m+1, p}\right) & |n|+|m|=2 k, k \in \mathbb{Z}, \\
-\left(\alpha_{1}-\alpha_{2}\right)\left(1+\varepsilon^{2} u_{n+1, m, p} u_{n, m+1, p}\right), & \\
\left(u_{n, m, p}-u_{n+1, m+1, p}\right)\left(u_{n+1, m, p}-u_{n, m+1, p}\right) & |n|+|m|=2 k+1, k \in \mathbb{Z}, \\
-\left(\alpha_{1}-\alpha_{2}\right)\left(1+\varepsilon^{2} u_{n, m, p} u_{n+1, m+1, p}\right), &
\end{array}\right. \\
& \bar{A}=\left\{\begin{array}{cl}
\left(u_{n, m, p+1}-u_{n+1, m+1, p+1}\right)\left(u_{n+1, m, p+1}-u_{n, m+1, p+1}\right) & |n|+|m|=2 k, k \in \mathbb{Z}, \\
-\left(\alpha_{1}-\alpha_{2}\right)\left(1+\varepsilon^{2} u_{n, m, p+1} u_{n+1, m+1, p+1}\right), & \\
\left(u_{n, m, p+1}-u_{n+1, m+1, p+1}\right)\left(u_{n+1, m, p+1}-u_{n, m+1, p+1}\right) & |n|+|m|=2 k+1, k \in \mathbb{Z}, \\
-\left(\alpha_{1}-\alpha_{2}\right)\left(1+\varepsilon^{2} u_{n+1, m, p+1} u_{n, m+1, p+1}\right), &
\end{array}\right.
\end{aligned}
$$

$$
B=\left\{\begin{array}{cl}
\left(u_{n, m, p}-u_{n, m+1, p+1}\right)\left(u_{n, m+1, p}-u_{n, m, p+1}\right) & |n|+|m|=2 k, k \in \mathbb{Z}, \\
-\left(\alpha_{2}-\alpha_{3}\right)\left(1+\varepsilon^{2} u_{n, m+1, p} u_{n, m, p+1}\right), & \\
\left(u_{n, m, p}-u_{n, m+1, p+1}\right)\left(u_{n, m+1, p}-u_{n, m, p+1}\right) & |n|+|m|=2 k+1, k \in \mathbb{Z}, \\
-\left(\alpha_{2}-\alpha_{3}\right)\left(1+\varepsilon^{2} u_{n, m, p} u_{n, m+1, p+1}\right), &
\end{array}\right.
$$

$$
\bar{B}=\left\{\begin{array}{cl}
\left(u_{n+1, m, p}-u_{n, m+1, p+1}\right)\left(u_{n+1, m+1, p}-u_{n+1, m, p+1}\right) & |n|+|m|=2 k, k \in \mathbb{Z}, \\
-\left(\alpha_{2}-\alpha_{3}\right)\left(1+\varepsilon^{2} u_{n+1, m, p} u_{n+1, m+1, p+1},\right. & \\
\left(u_{n+1, m, p}-u_{n+1, m+1, p+1}\right)\left(u_{n+1, m+1, p}-u_{n+1, m, p+1}\right) & |n|+|m|=2 k+1, k \in \mathbb{Z}, \\
-\left(\alpha_{2}-\alpha_{3}\right)\left(1+\varepsilon^{2} u_{n+1, m+1, p} u_{n+1, m, p+1}\right), &
\end{array}\right.
$$

$$
C=\left\{\begin{array}{cl}
\left(u_{n, m, p}-u_{n+1, m, p+1}\right)\left(u_{n+1, m, p}-u_{n, m, p+1}\right) & |n|+|m|=2 k, k \in \mathbb{Z}, \\
-\left(\alpha_{1}-\alpha_{3}\right)\left(1+\varepsilon^{2} u_{n+1, m, p} u_{n, m, p+1}\right), & \\
\left(u_{n, m, p}-u_{n+1, m, p+1}\right)\left(u_{n+1, m, p}-u_{n, m, p+1}\right) & |n|+|m|=2 k+1, k \in \mathbb{Z}, \\
-\left(\alpha_{1}-\alpha_{3}\right)\left(1+\varepsilon^{2} u_{n, m, p} u_{n+1, m, p+1}\right), &
\end{array}\right.
$$




$$
\bar{C}=\left\{\begin{array}{cl}
\left(u_{n, m+1, p}-u_{n+1, m+1, p+1}\right)\left(u_{n+1, m+1, p}-u_{n, m+1, p+1}\right) & |n|+|m|=2 k, k \in \mathbb{Z}, \\
-\left(\alpha_{1}-\alpha_{3}\right)\left(1+\varepsilon^{2} u_{n, m+1, p} u_{n+1, m+1, p+1}\right), & \\
\left(u_{n, m+1, p}-u_{n+1, m+1, p+1}\right)\left(u_{n+1, m+1, p}-u_{n, m+1, p+1}\right) & |n|+|m|=2 k+1, k \in \mathbb{Z}, \\
-\left(\alpha_{1}-\alpha_{2}\right)\left(1+\varepsilon^{2} u_{n+1, m+1, p} u_{n, m+1, p+1}\right), &
\end{array}\right.
$$

Up to now we showed how, given a CAC quad equation $Q$, it is possible to embed it into a partial difference equation in $\mathbb{Z}^{2}$ given by (A.7). Furthermore we showed that this procedure can be extended along the third dimension in such a way that the consistency is preserved. This have been done following [8] and filling the details (which are going to be important).

The partial difference equation (A.7) is not very manageable since we have to change equation according to the point of the lattice we are in. It will be more efficient to have an expression which "knows" by itself in which point we are. This can obtained by going over to non-autonomous equations as was done in the BW lattice case 31.

We shall present here briefly how from (A.7) it is possible to construct an equivalent non-autonomous system, and moreover how to construct the nonautonomous version of CAC (A.10).

We take an equation $\hat{Q}$ constructed by a linear combination of the equations (1.5) with $n$ and $m$ depending coefficients:

$$
\begin{aligned}
\widehat{Q} & =f_{n, m} Q\left(u_{n, m}, u_{n+1, m}, u_{n, m+1}, u_{n+1, m+1}\right)+ \\
& +\left|f_{n, m}\right| Q\left(u_{n, m}, u_{n+1, m}, u_{n, m+1}, u_{n+1, m+1}\right)+ \\
& +\underline{f}_{n, m} \underline{Q}\left(u_{n, m}, u_{n+1, m}, u_{n, m+1}, u_{n+1, m+1}\right)+ \\
& +\left|\underline{f}_{n, m}\right| \underline{Q}\left(u_{n, m}, u_{n+1, m}, u_{n, m+1}, u_{n+1, m+1}\right) .
\end{aligned}
$$

We require that it satisfies the following conditions:

1. The coefficients are periodic of period 2 in both directions, since, in the $\mathbb{Z}^{2}$ embedding, the elementary cell is a $2 \times 2$ one.

2. The coefficients are such that they produce the right equation in a given lattice point as specified in (A.7).

Condition 11 implies that any function $\tilde{f}_{n, m}$ in (A.14), i.e. either $f_{n, m}$ or $\mid f_{n, m}$ or $\underline{f}_{n, m}$ or $\underline{f}_{n, m}$, solves the two ordinary difference equations:

$$
\tilde{f}_{n+2, m}-\tilde{f}_{n, m}=0, \quad \tilde{f}_{n, m+2}-\tilde{f}_{n, m}=0,
$$

whose solution is:

$$
\tilde{f}_{n, m}=c_{0}+c_{1}(-1)^{n}+c_{2}(-1)^{m}+c_{3}(-1)^{n+m}
$$

with $c_{i}$ constants to be determined.

The condition 2 depends on the choice of the equation in (A.7) and will give some "boundary conditions" for the function $\tilde{f}$, allowing us to fix the coefficients $c_{i}$. For $f_{n, m}$, for example, we have, substituting the appropriate lattice points, the following conditions:

$$
f_{2 k, 2 k}=1, \quad f_{2 k+1,2 k}=f_{2 k, 2 k+1}=f_{2 k+1,2 k+1}=0,
$$


which yield:

$$
f_{n, m}=\frac{1+(-1)^{n}+(-1)^{m}+(-1)^{n+m}}{4} .
$$

In an analogous manner we obtain the form of the other functions in (A.14):

$$
\begin{aligned}
\mid f_{n, m} & =\frac{1-(-1)^{n}+(-1)^{m}-(-1)^{n+m}}{4}, \\
\underline{f}_{n, m} & =\frac{1+(-1)^{n}-(-1)^{m}-(-1)^{n+m}}{4}, \\
\underline{f}_{n, m} & =\frac{1-(-1)^{n}-(-1)^{m}+(-1)^{n+m}}{4} .
\end{aligned}
$$

Then inserting (A.18) in (A.14) we obtain a non-autonomous equation which corresponds to (A.7).

If the quad-equation $Q$ possess some discrete symmetries, the expression (A.14) greatly simplify. If an equation $Q$ is invariant under the discrete group $D_{4}$ we trivially have, using (1.6), $\widehat{Q}=Q$. This result states that an equation with the symmetry (1.6) is defined on a monochromatic lattice, as expected since we are in the case of the ABS classification [1. If the equation $Q$ has the symmetries of the rhombus, namely (1.8), we get:

$$
\widehat{Q}=\left(f_{n, m}+\mid \underline{f}_{n, m}\right) Q+\left(\mid f_{n, m}+\underline{f}_{n, m}\right) \mid Q
$$

and using (A.18):

$$
f_{n, m}+\left|\underline{f}_{n, m}=F_{n+m}^{(+)}, \quad\right| f_{n, m}+\underline{f}_{n, m}=F_{n+m}^{(-)} .
$$

where:

$$
F_{k}^{( \pm)}=\frac{1 \pm(-1)^{k}}{2}, \quad k \in \mathbb{Z} .
$$

This obviosly match with the results in 31 .

In the case of trapezoidal symmetry (1.10) one obtains:

$$
\widehat{Q}=\left(f_{n, m}+\mid f_{n, m}\right) Q+\left(\underline{f}_{n, m}+\mid \underline{f}_{n, m}\right) \underline{Q},
$$

with

$$
f_{n, m}+\left|f_{n, m}=F_{m}^{(+)}, \quad \underline{f}_{n, m}+\right| \underline{f}_{n, m}=F_{m}^{(-)},
$$

As an example of such construction let us consider again ${ }_{r} H_{1}^{\varepsilon}$ (A.1). Since we are in the rhombic case [31] we use formula (A.20) and get:

$$
\begin{aligned}
{ }_{r} \widehat{H}_{1}^{\varepsilon} & =\left(u_{n, m}-u_{n+1, m+1}\right)\left(u_{n+1, m}-u_{n, m+1}\right)-\left(\alpha_{1}-\alpha_{2}\right) \\
& +\left(\alpha_{1}-\alpha_{2}\right) \varepsilon^{2}\left(F_{n+m}^{(+)} u_{n+1, m} u_{n, m+1}+\mid F_{n+m}^{(-)} u_{n, m} u_{n+1, m+1}\right)=0
\end{aligned}
$$

which corresponds to the case $\sigma=1$ of 31 (the discussion of the meaning of parameter $\sigma$ in 31 is postponed to the end this Appendix).

The consistency of a generic system of quad equations is obtained by considering the consistency of the tilded equations as displayed in (A.11). We now construct, starting from (A.13), the non autonomous partial difference equations 
in the $(n, m)$ variables using the weights $\tilde{f}_{n, m}$, as given in (A.14), applied to the relevant equations. Carrying out such construction, we end with the following sextuple of equations:

$$
\begin{aligned}
& \widehat{A}\left(u_{n, m, p}, u_{n+1, m, p}, u_{n, m+1, p}, u_{n+1, m+1, p}\right)=f_{n, m} A+\left|f_{n, m}\right| A+ \\
& +\underline{f}_{n, m} \underline{A}+\left|\underline{f}_{n, m}\right| \underline{A}=0, \\
& \widehat{\bar{A}}\left(u_{n, m, p+1}, u_{n+1, m, p+1}, u_{n, m+1, p+1}, u_{n+1, m+1, p+1}\right)=f_{n, m} \bar{A}+\left|f_{n, m}\right| \bar{A}+ \\
& +\underline{f}_{n, m} \underline{\bar{A}}+\left|\underline{f}_{n, m}\right| \underline{\bar{A}}=0, \\
& \widehat{B}\left(u_{n, m, p}, u_{n, m+1, p}, u_{n, m, p+1}, u_{n, m+1, p+1}\right)=f_{n, m} B+\left|f_{n, m}\right| \bar{B}+ \\
& \text { (A.25c) } \\
& +\underline{f}_{n, m} \underline{B}+\left|\underline{f}_{n, m}\right| \underline{\bar{B}}=0, \\
& \widehat{\bar{B}}\left(u_{n+1, m, p}, u_{n+1, m+1, p}, u_{n+1, m, p+1}, u_{n+1, m+1, p+1}\right)=f_{n, m} \bar{B}+\left|f_{n, m}\right| B+ \\
& +\underline{f}_{n, m} \underline{\bar{B}}+\left|\underline{f}_{n, m}\right| \underline{B}=0, \\
& \widehat{C}\left(u_{n, m, p}, u_{n+1, m, p}, u_{n, m, p+1}, u_{n+1, m, p+1}\right)=f_{n, m} C+\left|f_{n, m}\right| C+ \\
& +\underline{f}_{n, m} \bar{C}+\left|\underline{f}_{n, m}\right| \bar{C}=0, \\
& \widehat{\bar{C}}\left(u_{n, m+1, p}, u_{n+1, m, p}, u_{n, m+1, p+1}, u_{n+1, m+1, p+1}\right)=f_{n, m} \bar{C}+\left|f_{n, m}\right| \bar{C}+ \\
& +\underline{f}_{n, m} C+\left|\underline{f}_{n, m}\right| C=0,
\end{aligned}
$$

where all the functions on the right hand side of the equality sign are evaluated on the point indicated on the left hand side.

We note that a Lax pair obtained by making use of equations A.25) will be effectively a pair, since the couples $(\widehat{B}, \widehat{\bar{B}})$ and $(\widehat{C}, \widehat{\bar{C}})$ are related by translation so they are just two different solutions of the same equation. Indeed by using the properties of the functions $\tilde{f}_{n, m}$ we have:

$$
\widehat{\bar{B}}=T_{n} \widehat{B}, \quad \widehat{\bar{C}}=T_{m} \widehat{C},
$$

where $T_{n}$ is the operator of translation in the $n$ direction, and $T_{m}$ the operator of translation in the $m$ direction. This allow us to construct Bäcklund transformations and Lax pair in the usual way [6,25].

As a final example we shall derive the non-autonomous side equations for $H_{1}^{\varepsilon}$ and its Lax pair. We will then confront the result with that obtained in [31. Considering A.12, A.25, A.26) and using the fact that the equation is rhombic A.20) we get the following result:

$$
\begin{aligned}
\widehat{A}= & \left(u_{n, m, p}-u_{n+1, m+1, p}\right)\left(u_{n+1, m, p}-u_{n, m+1, p}\right)-\left(\alpha_{1}-\alpha_{2}\right) . \\
& \cdot\left[1+\varepsilon^{2}\left(F_{n+m}^{(+)} u_{n+1, m, p} u_{n, m+1, p}+F_{n+m}^{(-)} u_{n, m, p} u_{n+1, m+1, p}\right)\right]=0,
\end{aligned}
$$




$$
\begin{aligned}
\widehat{\bar{A}}= & \left(u_{n, m, p+1}-u_{n+1, m+1, p+1}\right)\left(u_{n+1, m, p+1}-u_{n, m+1, p+1}\right)-\left(\alpha_{1}-\alpha_{2}\right) . \\
& \cdot\left[1+\varepsilon^{2}\left(F_{n+m}^{(-)} u_{n+1, m, p+1} u_{n, m+1, p+1}+F_{n+m}^{(+)} u_{n, m, p+1} u_{n+1, m+1, p+1}\right)\right]=0, \quad \text { (A.27b) } \\
\widehat{B}= & \left(u_{n, m, p}-u_{n, m+1, p+1}\right)\left(u_{n, m+1, p}-u_{n, m, p+1}\right)-\left(\alpha_{2}-\alpha_{3}\right) \cdot \\
& \cdot\left[1+\varepsilon^{2}\left(F_{n+m}^{(+)} u_{n, m+1, p} u_{n, m, p+1}+F_{n+m}^{(-)} u_{n, m, p} u_{n, m+1, p+1}\right)\right]=0, \\
\widehat{C}= & \left(u_{n, m, p}-u_{n+1, m, p+1}\right)\left(u_{n+1, m, p}-u_{n, m, p+1}\right)-\left(\alpha_{1}-\alpha_{3}\right) \cdot \\
& \cdot\left[1+\varepsilon^{2}\left(F_{n+m}^{(+)} u_{n+1, m, p} u_{n, m, p+1}+F_{n+m}^{(-)} u_{n, m, p} u_{n+1, m, p+1}\right)\right]=0,
\end{aligned}
$$

From the equations $\widehat{B}$ and $\widehat{C}$ we find, up to a sign and commond factor, which we are can eliminate since the Lax pair is defined from CAC only up to projective equivalence [11,23, the following Lax pair:

$$
\begin{aligned}
& L=\left(\begin{array}{cc}
u_{n, m} & \alpha_{1}-\alpha_{3}-u_{n, m} u_{n+1, m} \\
1 & -u_{n+1, m}
\end{array}\right) \\
& +\left(\alpha_{1}-\alpha_{3}\right) \varepsilon^{2}\left(\begin{array}{cc}
F_{n+m}^{(+)} u_{n+1, m} & 0 \\
0 & -F_{n+m}^{(-)} u_{n, m}
\end{array}\right) \\
& M=\left(\begin{array}{cc}
u_{n, m} & \alpha_{2}-\alpha_{3}-u_{n, m} u_{n, m+1} \\
1 & -u_{n, m+1}
\end{array}\right) \\
& +\left(\alpha_{2}-\alpha_{3}\right) \varepsilon^{2}\left(\begin{array}{cc}
F_{n+m}^{(+)} u_{n, m+1} & 0 \\
0 & -F_{n+m}^{(-)} u_{n, m}
\end{array}\right), \\
& \bar{L}=\left(\begin{array}{cc}
u_{n, m+1} & \alpha_{1}-\alpha_{3}-u_{n, m+1} u_{n+1, m+1} \\
1 & -u_{n+1, m+1}
\end{array}\right) \\
& +\left(\alpha_{1}-\alpha_{3}\right) \varepsilon^{2}\left(\begin{array}{cc}
F_{n+m}^{(-)} u_{n+1, m+1} & 0 \\
0 & -F_{n+m}^{(+)} u_{n, m+1}
\end{array}\right) \\
& \bar{M}=\left(\begin{array}{cc}
u_{n+1, m} & \alpha_{2}-\alpha_{3}-u_{n+1, m} u_{n+1, m+1} \\
1 & -u_{n+1, m+1}
\end{array}\right) \\
& +\left(\alpha_{2}-\alpha_{3}\right) \varepsilon^{2}\left(\begin{array}{cc}
F_{n+m}^{(-)} u_{n+1, m+1} & 0 \\
0 & -F_{n+m}^{(+)} u_{n+1, m}
\end{array}\right) .
\end{aligned}
$$

This is a Lax pair since $\bar{L}=T_{m} L$ and $\bar{M}=T_{n} M$. We find that such matrices give as compatibility the equation (A.1) and correspond to the following proportionality factor $\tau$ [11:

$$
\tau=\frac{1+\varepsilon^{2}\left(F_{n+m}^{(-)} u_{n, m}+F_{n+m}^{(+)} u_{n+1, m}\right)}{1+\varepsilon^{2}\left(F_{n+m}^{(-)} u_{n, m}+F_{n+m}^{(+)} u_{n, m+1}\right)} .
$$

The Lax pair (A.28) is Gauge equivalent to that obtained in [31] with gauge:

$$
G=\left(\begin{array}{cc}
0 & 1 \\
-1 & 0
\end{array}\right) .
$$


A similar calculation could be done for the other two rhombic equations, and, up to gauge transformations, will give the same result. Indeed the gauge transformations (A.30) is needed for $H_{1}^{\varepsilon}$ and $H_{2}^{\varepsilon}$ whereas for $H_{3}^{\varepsilon}$ we need the gauge:

$$
\tilde{G}=\left(\begin{array}{cc}
0 & (-1)^{n+m} \\
-(-1)^{n+m} & 0
\end{array}\right) .
$$

We end this Appendix with the following remark: at the level of the nonautonomous equations the choice of origin of $\mathbb{Z}^{2}$ in a point different from $x$ in (A.7) would have led to different initial conditions in (A.17), which ultimately lead to the following form for the functions $f$ :

$$
\begin{aligned}
f_{n, m}^{\sigma_{1}, \sigma_{2}} & =\frac{1+\sigma_{1}(-1)^{n}+\sigma_{2}(-1)^{m}+\sigma_{1} \sigma_{2}(-1)^{n+m}}{4}, \\
\mid f_{n, m}^{\sigma_{1}, \sigma_{2}} & =\frac{1-\sigma_{1}(-1)^{n}+\sigma_{2}(-1)^{m}-\sigma_{1} \sigma_{2}(-1)^{n+m}}{4}, \\
\underline{f}_{n, m}^{\sigma_{1}, \sigma_{2}} & =\frac{1+\sigma_{1}(-1)^{n}-\sigma_{2}(-1)^{m}-\sigma_{1} \sigma_{2}(-1)^{n+m}}{4}, \\
\underline{f}_{n, m}^{\sigma_{1}, \sigma_{2}} & =\frac{1-\sigma_{1}(-1)^{n}-\sigma_{2}(-1)^{m}+\sigma_{1} \sigma_{2}(-1)^{n+m}}{4},
\end{aligned}
$$

where the two constants $\sigma_{i} \in\{ \pm 1\}$ depends on the point chosen. Indeed if the point is $x$ we have $\sigma_{1}=\sigma_{2}=1$, whereas if we choose $x_{1}$ we have $\sigma_{1}=1$, $\sigma_{2}=-1$, if we choose $x_{2}$ then $\sigma_{1}=-1$ and $\sigma_{2}=1$ and finally if we choose $x_{12}$ we shall put $\sigma_{1}=\sigma_{2}=-1$. It is easy to see that in the rhombic and in the trapezoidal case the functions (A.32) collapse to the $\sigma$ version of the functions $F_{k}^{( \pm)}$as given by (A.21):

$$
F_{k}^{( \pm, \sigma)}=\frac{1 \pm \sigma(-1)^{k}}{2} .
$$

The final equation will then depend on $\sigma_{1}$ or $\sigma_{2}$, only if rhombic or trapezoidal.

It was proved in 31 that the transformations:

$$
v_{n, m}=u_{n+1, m}, \quad w_{n, m}=u_{n, m+1},
$$

map a rhombic equation with a certain $\sigma$ into the same rhombic equation with $-\sigma$. In 31 this fact was used to construct a Lax Pair and Bäcklund transformations. An analogous result can be easily proven for trapezoidal equations: using the transformation $w_{n, m}=u_{n, m+1}$ we can send a trapezoidal equation with a certain $\sigma$ into the same equation with $-\sigma$. A similar trasformation in the $n$ direction would just trivally leave invariant the trapezoidal equation, since there is no explicit dependence on $n$. However in general, if an equation does not posses discrete symmetries, as it is the case for a $H^{6}$ equation, no trasformation like A.34 would take the equation into itself with different coefficents. We can anyway construct a Lax pair with the procedure explained above, which is then slightly more general than the approach based on the transformations (A.34). 


\section{References}

[1] V. E. Adler, A. I. Bobenko, Yu. B. Suris Classification of integrable equations on quad-graphs. The consistency approach, Comm. Math. Phys. 233, 513543 (2003).

[2] V. E. Adler, A. I. Bobenko, Yu. B. Suris Discrete nonlinear hyperbolic equations. Classification of integrable cases, Funct. Anal. Appl. 43, 317 (2009).

[3] V. E. Adler, A. I. Bobenko and Yu. B. Suris, Discrete nonlinear hyperbolic equations. Classification of integrable cases, Funct. Anal. Apll. 43 (2009) $3-17$.

[4] V. E. Adler, A. I. Bobenko and Yu. B. Suris, Classification of the integrable discrete equations of the octahedron type, Intern. Math. Research Notices 60 (2011) 363-401.

[5] J. Atkinson, Linear quadrilateral lattice equations and multidimensional consistency, J. Phys. A: Math. Theor. 42, 454005 (2009).

[6] A. I. Bobenko, P. Schröder, J. M. Sullivan and G. M. Ziegler, Discrete differential geometry, Birkhäuser Verlag AG 2008.

[7] A.I. Bobenko, Yu.B. Suris, Integrable systems on quad-graphs, Int. Math. Res. Notices 11, 573611 (2002).

[8] R. Boll, Classification of $3 D$ consistent quad-equations, J. Nonl. Math. Phys. 18, no. 3, 337-365 (2011).

[9] R. Boll, Corrigendum Classification of $3 D$ consistent quad-equations, $J$. Nonl. Math. Phys. 19, no. 4, 1292001 (2012).

[10] R. Boll, Classification and Lagrangian structure od $3 D$ consistent quadequations, Ph. D. dissertation (2012).

[11] T. Bridgman, W. Hereman, G. R. W. Quispel, P. H. van der Kamp, Symbolic computation of Lax pairs of partial difference equations using consistency around the cube, Found. Comput. Math. 13, no. 4, 517-544 (2013).

[12] S. Butler and Mike Hay, Two definitions of fake Lax pairs, AIP Conf. Proc. 1648, 180006 (2015).

[13] S. Butler, M. Hay, Simple identification of fake Lax pairs, arXiv:1311.2406.

[14] F. Calogero, A. Degasperis, Spectral Transform and Solitons I, Norht Holland Publishing Co. Amsterdam 1982.

[15] B. Grammaticos, A. Ramani, C. Viallet, Solvable Chaos, Phys. Lett. A 336 (2005) 152-158.

[16] G. Gubbiotti PhD thesis to be discussed in 2017.

[17] G. Gubbiotti and M. Hay, A SymPy module to calculate algebraic entropy for difference equations and quadrilateral partial difference equations, 2015, in preparation. 
[18] G. Gubbiotti, D. Levi and C. Scimiterna, On Partial Differential and Difference Equations with Symmetries Depending on Arbitrary Functions, Acta Polytechnica in press.

[19] G. Gubbiotti, C. Scimiterna and D. Levi, Linearizability and fake Lax pair for a consistent around the cube nonlinear non-autonomous quad-graph equation, Theor. Math. Phys. in press.

[20] J. Hietarinta, A new two-dimensional lattice model that is 'consistent around the cube', J. Phys. A: Math. Gen. 37, L67-L73 (2004).

[21] J. Hietarinta, Searching for CAC-maps, J. Nonl. Math. Phys. 12, no. 2, 223-230 (2005).

[22] J. Hietarinta and C. Viallet, Searching for integrable lattice maps using factorization, J. Phys. A: Math. Theor. 40 (2007) 12629-12643.

[23] J. Hietarinta, C. Viallet, Weak Lax pairs for lattice equations, Nonlin. 25, 1955-1966 (2012).

[24] F.W. Nijhoff, Lax pair for the Adler (lattice Krichever- Novikov) system, Phys. Lett. A 297, 4958 (2002).

[25] F.W. Nijhoff, A. Walker, The discrete and continuous Painlevé VI hierarchy and the Garnier systems, Glasgow Math. J. 43A, 109 (2001)

[26] A. Ramani, N. Joshi, B. Grammaticos, T. Tamizhmani, Deconstructing an integrable lattice equation, J. Phys. A: Math. Gen. 39, L145-L149 (2006).

[27] SymPy: Python library for symbolic mathematics, SymPy Development Team, http://www . sympy .org, (2014)

[28] Tremblay, S. and Grammaticos, B. and Ramani, A., Integrable lattice equations and their growth properties, Phys. Lett. A 278 (2001) 319-324.

[29] C. Viallet, Algebraic Entropy for lattice equations, 2006, arXiv:math-ph/0609043.

[30] C. Viallet, On the algebraic structure of rational discrete dynamical systems, J. Phys. A: Math. Theor. 48 (2015) 16FT01 (21pp), arXiv:1501.06384 [nlin.SI].

[31] P. D. Xenitidis, V. G. Papageorgiou, Symmetries and integrability of discrete equations defined on a black-white lattice, J. Phys. A: Math. Theor. 42, 454025 (2009).

[32] R.I. Yamilov, Symmetries as integrability criteria for differential difference equations, J. Phys. A: Math. Gen. 39 (2006) R541-R623. 